\title{
Semiclassical approach to ground-state properties of hard-core bosons in two dimensions
}

\author{
Tommaso Coletta, ${ }^{1}$ Nicolas Laflorencie, ${ }^{2}$ and Frédéric Mila ${ }^{1}$ \\ ${ }^{1}$ Institute of Theoretical Physics, Ecole Polytechnique Fédérale de Lausanne (EPFL), CH-1015 Lausanne, Switzerland \\ ${ }^{2}$ Laboratoire de Physique Théorique, Université de Toulouse, UPS, (IRSAMC), F-31062 Toulouse, France
}

(Received 23 December 2011; revised manuscript received 5 March 2012; published 30 March 2012)

\begin{abstract}
Motivated by some inconsistencies in the way quantum fluctuations are included beyond the classical treatment of hard-core bosons on a lattice in the recent literature, we revisit the large- $S$ semiclassical approach to hard-core bosons on the square lattice at $T=0$. First of all, we show that, if one stays at the purely harmonic level, the only correct way to get the $1 / S$ correction to the density is to extract it from the derivative of the ground-state energy with respect to the chemical potential, and that to extract it from a calculation of the ground-state expectation value of the particle number operator, it is necessary to include $1 / \sqrt{S}$ corrections to the harmonic ground state. Building on this alternative approach to get $1 / S$ corrections, we provide the first semiclassical derivation of the momentum distribution, and we revisit the calculation of the condensate density. The results of these as well as other physically relevant quantities such as the superfluid density are systematically compared to quantum Monte Carlo simulations. This comparison shows that the logarithmic corrections in the dilute Bose gas limit are only captured by the semiclassical approach if the $1 / S$ corrections are properly calculated, and that the semiclassical approach is able to reproduce the $1 / k$ divergence of the momentum distribution at $k=0$. Finally, the effect of $1 / S^{2}$ corrections is briefly discussed.
\end{abstract}

DOI: 10.1103/PhysRevB.85.104421

PACS number(s): 05.30.Jp

\section{INTRODUCTION}

Models of interacting bosons on a lattice are ubiquitous. They have been introduced to describe the low-energy physics of systems as different as thin superconducting films, ${ }^{1}$ Josephson junction arrays, ${ }^{2}{ }^{4} \mathrm{He}$ on substrates, ${ }^{3-5}$ cold atoms in optical lattices, ${ }^{6}$ bipolarons, ${ }^{7}$ or quantum magnets in a field. ${ }^{8,9}$ In simple (unfrustrated) geometries, quantum Monte Carlo (QMC) simulations do not suffer from the minus sign problem, and the resulting picture is often quite clear. ${ }^{10}$ However, in many recent applications, the relevant effective model contains terms that lead to a severe minus sign problem. This is, for example, true for the bosonic description of frustrated quantum magnets in a magnetic field, ${ }^{11}$ where QMC approaches are not appropriate. To investigate such models, it is important to develop alternative approaches.

A very important subclass is that of models of hard-core bosons on a lattice in which the on-site repulsion is assumed to be infinite so that it is impossible to have more than one boson at a given site. Such models appear, for instance, very naturally in the description of dimer-based spin- $1 / 2$ quantum magnets in a field. ${ }^{9}$ In this paper, we will concentrate on a model of hard-core bosons on a two-dimensional square lattice described by the simple Hamiltonian:

$$
\mathcal{H}=-t \sum_{\langle i, j\rangle}\left(a_{i}^{\dagger} a_{j}+a_{i} a_{j}^{\dagger}\right)-\mu \sum_{i} n_{i}
$$

where $t$ is the hopping amplitude between neighboring sites, $\mu$ is the chemical potential, and $a_{i}^{\dagger}\left(a_{i}\right)$ denotes the operator creating (destroying) a hard-core boson at site $i$. One distinct property of hard-core boson models is that they can be mapped exactly onto spin-1/2 models using the Matsuda-Matsubara transformation: ${ }^{3} n_{i}=S_{i}^{z}+1 / 2, a_{i}^{\dagger}=S_{i}^{+}$, and $a_{i}=S_{i}^{-}$. The equivalent spin-1/2 model is a ferromagnetic $X Y$ model with magnetic field $\mu$ pointing in the $z$ direction:

$$
\mathcal{H}=-t \sum_{\langle i, j\rangle} 2\left(S_{i}^{x} S_{j}^{x}+S_{i}^{y} S_{j}^{y}\right)-\mu \sum_{i}\left(S_{i}^{z}+1 / 2\right) .
$$

On the basis of this mapping, a semiclassical approximation can be developed starting from the large $S$ limit of this spin Hamiltonian. This approach has been developed in a series of papers. ${ }^{12-14}$ The paper by Bernardet and coworkers ${ }^{14}$ includes a careful comparison with QMC simulations and shows that, already at the order of linear-spin wave theory, the semiclassical approach is quantitatively accurate.

Building on this success, this semiclassical approach has recently been used quite systematically in the investigation of frustrated models ${ }^{13,15,17-21}$ for which it is often the only available analytical approximation. These studies have revealed a number of subtleties, however, in the implementation of the semiclassical approximation. A recurrent problem concerns the calculation of the bosonic density as a function of the chemical potential, ${ }^{22,23}$ or equivalently of the magnetization as a function of the field. Bernardet et al. have calculated the density as the opposite of the derivative of the energy with respect to the chemical potential, which is equivalent to calculating the magnetization as the opposite of the derivative of the energy with respect to the field. But one could, in principle, equally well calculate the magnetization as the expectation value of the operator $S^{z}$ in the ground state. However, at the harmonic level, the two definitions do not lead to the same answer, and it is not clear which definition should be preferred. In addition, in its current setting, the semiclassical approach only allows to calculate in a systematic way quantities that can be derived from the ground-state energy, i.e., the density and the superfluid stiffness. For instance, no attempt has been made so far to calculate other ground-state properties such as the momentum distribution function. Finally, no attempt to check the convergence of 
the $1 / S$ expansion by calculating higher-order corrections has been made.

In the present paper, we address all these issues. First of all, we show that, to get the same result using the two definitions of the density, one has to include $1 / \sqrt{S}$ corrections to the harmonic ground state. These corrections have to be included to get the correct answer to order $1 / S$ because they contribute at this order when calculating the expectation value of $S^{z}$. In the low-density limit, we also show that these corrections are crucial to get the logarithmic corrections predicted long ago for interacting two-dimensional bosons. Building on this success, we use this corrected ground state to calculate the momentum distribution function, and we show that it leads to a divergence at zero momentum that agrees with QMC results. We also provide two complementary ways to calculate the condensate at the order $1 / S$ : from the derivative of the energy with respect to a transverse field, and by a calculation to the zero-momentum occupation factor using the perturbed ground state. Finally, we calculate the $1 / S^{2}$ correction to the ground-state energy and show that it improves over the $1 / S$ result, supporting the basic assumption of the semiclassical approach that the $1 / S$ expansion is well behaved even for $S=1 / 2$.

Let us emphasize that we agree with all the results of Ref. 14 to order $1 / S$. In that respect, the main objective of the present paper is to show how these results can be obtained from perturbing the harmonic ground state with two new results: a clear answer regarding the appropriate way to calculate the expectation value of observables at the order $1 / S$, and the first semiclassical calculation of the momentum distribution.

This paper is organized as follows. In Sec. II, the model is treated in the context of linear spin wave theory (LSW). Section III is devoted to the semiclassical correction of the harmonic ground state and to the computation of several observables in this perturbed ground state. Section IV presents a comparison of the spin-wave results obtained with the results of QMC simulations. Section V discusses the validity in the context of a $1 / S$ expansion of the sum rule that states that the total density is equal to the sum of the condensate density and of the average momentum distribution function. Section VI presents some results obtained beyond the linear spin wave approximation. A short conclusion is given in Sec. VII. Finally, some details about the calculation of the superfluid density and of the momentum distribution are given in Appendices A and $\mathrm{B}$.

\section{LINEAR SPIN WAVE THEORY}

\section{A. The model}

To perform a semiclassical expansion, it will prove useful to extend the model of Eq. (1.2) in two ways. First of all, we rescale the amplitudes in such a way that the various terms are of the same order in the large $S$ limit while the Hamiltonian of Eq. (1.2) is recovered for $S=1 / 2$. Secondly, and more importantly, we introduce a transverse field $\Gamma \geqslant 0$ in the $x$ direction. These modifications lead to the Hamiltonian

$\mathcal{H}=-\frac{t}{S^{2}} \sum_{\langle i, j\rangle}\left(S_{i}^{x} S_{j}^{x}+S_{i}^{y} S_{j}^{y}\right)-\frac{\mu}{S} \sum_{i} S_{i}^{z}-\frac{\Gamma}{S} \sum_{i} S_{i}^{x}$.
The introduction of a transverse field $\Gamma$ turned out to be an essential ingredient in two respects. On one hand, it allows one to calculate the condensate density as the opposite of the derivative of the ground-state energy with respect to $\Gamma$, hence to get an expression that is correct to order $1 / S$. On the other hand, it breaks the continuous U(1) symmetry of the Hamiltonian of Eq. (1.2) and opens a gap in the spectrum of the model. Thanks to this gap, the correction to the harmonic ground state is not divergent, and the corrected ground state can be used to calculate the expectation value of various observables to order $1 / S$. The results for the original model are then obtained by taking the limit $\Gamma \rightarrow 0$ of the expectation values.

\section{B. Classical solution}

In the classical limit, spin operators are replaced by threedimensional vectors of norm $S$. In the absence of a transverse field, the ground state consists of spins ordered ferromagnetically in the $x-y$ plane with a longitudinal magnetization $m$ that varies linearly with the magnetic field $\mu$ until saturation. When $\Gamma>0$, the classical solution lies in the $x-z$ plane and can be parametrized as follows:

$$
\left(\begin{array}{c}
S_{i}^{x} \\
S_{i}^{y} \\
S_{i}^{z}
\end{array}\right)=S\left(\begin{array}{c}
\sin \theta \\
0 \\
\cos \theta
\end{array}\right) .
$$

With this parametrization, the classical energy per site is given by

$$
E^{(0)}=-2 t \sin ^{2} \theta-\mu \cos \theta-\Gamma \sin \theta .
$$

The angle $\theta$ is fixed by minimizing the classical energy,

$$
-4 t \sin \theta \cos \theta+\mu \sin \theta-\Gamma \cos \theta=0 .
$$

In the limit $\Gamma \rightarrow 0$, Eq. (2.4) has the simple solution $\cos \theta_{0}=$ $\mu / 4 t$, and $S_{i}^{x}$ is different from zero in the field range $-4 \leqslant$ $\mu / t \leqslant 4$. This defines the critical chemical potential $\mu_{c}=-4 t$ at which the system starts to acquire a transverse magnetization. For $S=1 / 2$, at $\mu>\mu_{c}$, we have $S_{i}^{z}>-1 / 2$, which in terms of the original hardcore boson model corresponds to a nonzero density of bosons. Thus $\mu_{c}$ is the value of chemical potential at which a hardcore boson population starts to develop. For $\Gamma \neq 0$, the angle $\theta$ is a function of $\Gamma$. In the following, we will focus on small $\Gamma$ case, and we will calculate the small $\Gamma$ correction to several quantities. From the equation $\sin \theta(-4 t \cos \theta+\mu)=\Gamma \cos \theta$, it is easy to see that the first-order correction to $\theta$ is given by

$$
\left.\frac{\partial \theta}{\partial \Gamma}\right|_{\Gamma=0}=\frac{\cos \theta_{0}}{4 t \sin ^{2} \theta_{0}} .
$$

The classical onsite magnetization is $S^{z}=S \cos \theta$ and the classical hardcore boson density is given by $\rho^{\text {class }}=(\cos \theta+$ 1)/2. In the limit where the transverse field vanishes, the classical density is given by

$$
\rho^{\text {class }}=\frac{1}{2}\left(\frac{\mu}{4 t}+1\right) \text {. }
$$




\section{Holstein-Primakoff transformation}

In order to study the effect of quantum fluctuations around this classical solution, we start by performing a rotation of the spins at each site:

$$
\begin{aligned}
& S_{i}^{x}=\cos \theta S_{i}^{x^{\prime}}+\sin \theta S_{i}^{z^{\prime}}, \\
& S_{i}^{y}=S_{i}^{y^{\prime}}, \\
& S_{i}^{z}=-\sin \theta S_{i}^{x^{\prime}}+\cos \theta S_{i}^{z^{\prime}},
\end{aligned}
$$

such that the Hamiltonian of Eq. (2.1), expressed in the rotated frame $\left(x^{\prime}, y^{\prime}, z^{\prime}\right)$, has a ferromagnetic ground state. The new spin operators can be expressed in terms of Holstein-Primakoff bosons. ${ }^{24}$ To next to leading order, the expressions take the following form:

$$
\begin{aligned}
S_{i}^{z^{\prime}} & =S-b_{i}^{\dagger} b_{i}, \\
S_{i}^{x^{\prime}} & =\frac{\sqrt{2 S}}{2}\left(b_{i}+b_{i}^{\dagger}\right)-\frac{1}{4 \sqrt{2 S}}\left(n_{i} b_{i}+b_{i}^{\dagger} n_{i}\right)+\cdots, \\
S_{i}^{y^{\prime}} & =\frac{\sqrt{2 S}}{2 i}\left(b_{i}-b_{i}^{\dagger}\right)-\frac{1}{4 i \sqrt{2 S}}\left(n_{i} b_{i}-b_{i}^{\dagger} n_{i}\right)+\cdots .
\end{aligned}
$$

The resulting Hamiltonian in terms of Holstein-Primakoff bosons can be expanded as

$$
\mathcal{H}=\sum_{n \geqslant 0} \mathcal{H}^{(n)},
$$

where $\mathcal{H}^{(n)}$ is proportional to $S^{-\frac{n}{2}}$. The first term of this series is $\mathcal{H}^{(0)}=N E^{(0)}, N$ being the total number of sites. By construction, $\mathcal{H}^{(1)}=0$ since we expand around a spin configuration, which is a classical minimum of the energy. $\mathcal{H}^{(2)}$ is quadratic in bosonic operators, while $\mathcal{H}^{(3)}$ and $\mathcal{H}^{(4)}$ contain only three or four boson terms, respectively. Their expressions are given by

$$
\begin{aligned}
\mathcal{H}^{(2)}=- & \frac{t}{2 S} \sum_{\langle i, j\rangle}\left(\cos ^{2} \theta+1\right)\left(b_{i} b_{j}^{\dagger}+b_{i}^{\dagger} b_{j}\right) \\
& -\frac{t}{2 S} \sum_{\langle i, j\rangle}\left(\cos ^{2} \theta-1\right)\left(b_{i} b_{j}+b_{i}^{\dagger} b_{j}^{\dagger}\right) \\
& +\frac{1}{S} \sum_{i} b_{i}^{\dagger} b_{i}\left(4 t \sin ^{2} \theta+\mu \cos \theta+\Gamma \sin \theta\right) \\
\mathcal{H}^{(3)} & =\frac{2 t}{S \sqrt{2 S}} \sum_{\langle i, j\rangle} n_{i}\left(b_{j}+b_{j}^{\dagger}\right) \sin \theta \cos \theta \\
\mathcal{H}^{(4)}=- & \frac{t}{S^{2}} \sum_{\langle i, j\rangle} \frac{1}{8}\left(1-\cos ^{2} \theta\right)\left(\left[n_{i}+n_{j}\right] b_{i} b_{j}+\text { H.c. }\right) \\
- & \frac{t}{S^{2}} \sum_{\langle i, j\rangle}-\frac{1}{8}\left(1+\cos ^{2} \theta\right)\left(b_{i}^{\dagger}\left[n_{i}+n_{j}\right] b_{j}+\text { H.c. }\right) \\
& -\frac{t}{S^{2}} \sum_{\langle i, j\rangle} \sin ^{2} \theta n_{i} n_{j} .
\end{aligned}
$$

The calculation of $1 / S$ corrections, to which most of the paper is devoted, is based on $\mathcal{H}^{(2)}$ and $\mathcal{H}^{(3)}$. The fourth-order correction $\mathcal{H}^{(4)}$ will only be used in Sec. VI when we calculate the $1 / S^{2}$ correction to the energy.

\section{Diagonalization of the harmonic Hamiltonian}

In terms of the Fourier transformations of the HolsteinPrimakoff operators defined by

$$
\begin{aligned}
& b_{j}=\frac{1}{\sqrt{N}} \sum_{\mathbf{k}} b_{\mathbf{k}} e^{i \mathbf{r}_{j} \mathbf{k}}, \quad b_{j}^{\dagger}=\frac{1}{\sqrt{N}} \sum_{\mathbf{k}} b_{\mathbf{k}}^{\dagger} e^{-i \mathbf{r}_{j} \mathbf{k}}, \\
& b_{\mathbf{k}}=\frac{1}{\sqrt{N}} \sum_{j} b_{j} e^{-i \mathbf{r}_{j} \mathbf{k}}, \quad b_{\mathbf{k}}^{\dagger}=\frac{1}{\sqrt{N}} \sum_{j} b_{j}^{\dagger} e^{i \mathbf{r}_{j} \mathbf{k}},
\end{aligned}
$$

$\mathcal{H}^{(2)}$ can be decoupled into a sum over different modes:

$$
\begin{aligned}
\mathcal{H}^{(2)}= & \frac{1}{S} \sum_{\mathbf{k}}\left(b_{\mathbf{k}}^{\dagger}, b_{-\mathbf{k}}\right)\left(\begin{array}{cc}
A_{\mathbf{k}} & B_{\mathbf{k}} \\
B_{\mathbf{k}} & A_{\mathbf{k}}
\end{array}\right)\left(\begin{array}{c}
b_{\mathbf{k}} \\
b_{-\mathbf{k}}^{\dagger}
\end{array}\right) \\
& -\frac{1}{2 S} \sum_{\mathbf{k}}\left(4 t \sin ^{2} \theta+\mu \cos \theta+\Gamma \sin \theta\right),
\end{aligned}
$$

where the coefficients $A_{\mathbf{k}}$ and $B_{\mathbf{k}}$ are defined by

$$
\begin{aligned}
A_{\mathbf{k}} & =-\frac{t}{2} \gamma_{\mathbf{k}}\left(\cos ^{2} \theta+1\right)+2 t \sin ^{2} \theta+\frac{\mu}{2} \cos \theta+\frac{\Gamma}{2} \sin \theta, \\
B_{\mathbf{k}} & =\frac{t}{2} \gamma_{\mathbf{k}} \sin ^{2} \theta,
\end{aligned}
$$

with $\gamma_{\mathbf{k}}=\cos k_{x}+\cos k_{y}$. With the help of Eq. (2.5), these coefficients can easily be expanded to linear order in $\Gamma$ :

$$
\begin{aligned}
& A_{\mathbf{k}} \approx A_{\mathbf{k}}^{0}+\frac{\Gamma}{2}\left(\frac{\cos ^{2} \theta_{0}}{\sin \theta_{0}} \frac{\gamma_{\mathbf{k}}}{2}+\frac{1}{\sin \theta_{0}}\right), \\
& B_{\mathbf{k}} \approx B_{\mathbf{k}}^{0}+\Gamma \frac{\cos ^{2} \theta_{0}}{4 \sin \theta_{0}} \gamma_{\mathbf{k}},
\end{aligned}
$$

where $A_{\mathbf{k}}^{0}=-t\left[\gamma_{\mathbf{k}}\left(1+\cos ^{2} \theta_{0}\right)-4\right] / 2$ and $B_{\mathbf{k}}^{0}=$ $\left(t \gamma_{\mathbf{k}} \sin ^{2} \theta_{0}\right) / 2$ denote the coefficients $A_{\mathbf{k}}$ and $B_{\mathbf{k}}$ in the absence of a transverse field. ${ }^{14}$ The second term in Eq. (2.14) can also be expanded to first order in $\Gamma$, leading to $-(1 / S)\left[\sum_{\mathbf{k}} 2 t+\Gamma /\left(2 \sin \theta_{0}\right)\right]$.

The quadratic Hamiltonian (2.14) can be diagonalized via a Bogoliubov transformation:

$$
b_{\mathbf{k}}=u_{\mathbf{k}} \alpha_{\mathbf{k}}-v_{\mathbf{k}} \alpha_{-\mathbf{k}}^{\dagger}, \quad b_{\mathbf{k}}^{\dagger}=u_{\mathbf{k}} \alpha_{\mathbf{k}}^{\dagger}-v_{\mathbf{k}} \alpha_{-\mathbf{k}} .
$$

The coefficients which ensure that the operators $\alpha_{\mathbf{k}}\left(\alpha_{\mathbf{k}}^{\dagger}\right)$ satisfy bosonic commutation relations and that the Hamiltonian is diagonal are given by

$$
\begin{aligned}
& u_{\mathbf{k}}^{2}=\frac{1}{2}\left(\frac{A_{\mathbf{k}}}{\sqrt{A_{\mathbf{k}}^{2}-B_{\mathbf{k}}^{2}}}+1\right), \\
& v_{\mathbf{k}}^{2}=\frac{1}{2}\left(\frac{A_{\mathbf{k}}}{\sqrt{A_{\mathbf{k}}^{2}-B_{\mathbf{k}}^{2}}}-1\right) .
\end{aligned}
$$

In terms of the Bogoliubov operators, and to first order in $\Gamma$, the Hamiltonian takes the diagonal form:

$$
\begin{aligned}
\mathcal{H}^{(2)}= & \frac{2}{S} \sum_{\mathbf{k}} \sqrt{A_{\mathbf{k}}^{2}-B_{\mathbf{k}}^{2}} \alpha_{\mathbf{k}}^{\dagger} \alpha_{\mathbf{k}} \\
& +\frac{1}{S} \sum_{\mathbf{k}}\left(\sqrt{A_{\mathbf{k}}^{2}-B_{\mathbf{k}}^{2}}-2 t-\frac{\Gamma}{2 \sin \theta_{0}}\right) .
\end{aligned}
$$


The ground state of the harmonic Hamiltonian $\mathcal{H}^{(2)}$ is the vacuum of $\alpha$ particles. We will refer to it as the harmonic ground state in the rest of this paper. The first-order $1 / S$ correction to the energy per site is given by

$$
E^{(2)}=\frac{1}{S N} \sum_{\mathbf{k}}\left(\sqrt{A_{\mathbf{k}}^{2}-B_{\mathbf{k}}^{2}}-2 t-\frac{\Gamma}{2 \sin \theta_{0}}\right) .
$$

The only difference with the approach of Ref. 14 is that, as long as the transverse field is strictly positive, the Bogoliubov transformation is well behaved even at $\mathbf{k}=0$ since the excitation spectrum is gapped. Indeed, for small $\mathbf{k}$ and $\Gamma$, the excitation energy $\Omega_{\mathbf{k}}=2 \sqrt{A_{\mathbf{k}}^{2}-B_{\mathbf{k}}^{2}} / S$ can be written as

$$
\Omega_{\mathbf{k}} \approx \sqrt{\Delta^{2}+v^{2} \mathbf{k}^{2}}
$$

with

$$
\Delta=\frac{2}{S} \sqrt{\Gamma t \sin \theta_{0}}+\mathcal{O}\left(\Gamma^{\frac{3}{2}}\right)
$$

and

$$
v=\frac{2}{S} t \sin \theta_{0}+\frac{\Gamma\left(1+3 \cos ^{2} \theta_{0}\right)}{4 S \sin ^{2} \theta_{0}}+\mathcal{O}\left(\Gamma^{2}\right) .
$$

In the limit $\Gamma \rightarrow 0$, the spectrum becomes gapless and linear, as expected for phononlike excitations in a superfluid.

\section{E. Calculation of the densities from the ground-state energy}

A system of bosons is characterized by three densities: the total density, the condensate density, and the superfluid density. They can all be calculated as derivatives of the ground-state energy. Using the Hellman-Feynman theorem that states that

$$
\left\langle\frac{\partial \mathcal{H}(h)}{\partial h}\right\rangle=\frac{\partial}{\partial h}\langle\mathcal{H}(h)\rangle,
$$

where $h$ is some parameter of the Hamiltonian, one can calculate the longitudinal magnetization $m$ as

$$
m(S)=-S \frac{\partial E^{(2)}(\Gamma=0)}{\partial \mu}
$$

and the transverse magnetization $m_{\perp}$ as

$$
m_{\perp}(S)=-\left.S \frac{\partial E^{(2)}}{\partial \Gamma}\right|_{\Gamma=0}
$$

while the spin stiffness is given by the second derivative of the energy with respect to a twist (see Appendix A). The advantage of deriving these densities from the ground-state energy is that, once we have an expression of the energy to a given order in $1 / S$, we obtain expressions of the densities that are correct at the same order. Let us discuss the result for the various densities.

\section{Total density}

Using the expression of the energy of Eq. (2.20) for $\Gamma=$ 0 , the derivative with respect to $\mu$ leads to the longitudinal magnetization

$$
m(S)=S \cos \theta_{0}+\frac{\cos \theta_{0}}{4} \frac{1}{N} \sum_{\mathbf{k}} \gamma_{\mathbf{k}} \sqrt{\frac{A_{\mathbf{k}}^{0}-B_{\mathbf{k}}^{0}}{A_{\mathbf{k}}^{0}+B_{\mathbf{k}}^{0}}} .
$$

The total density $\rho$ is related to the longitudinal magnetization by $\rho=m(S=1 / 2)+1 / 2$, which leads to

$$
\rho=\frac{1+\cos \theta_{0}}{2}+\frac{\cos \theta_{0}}{4} \frac{1}{N} \sum_{\mathbf{k}} \gamma_{\mathbf{k}} \sqrt{\frac{A_{\mathbf{k}}^{0}-B_{\mathbf{k}}^{0}}{A_{\mathbf{k}}^{0}+B_{\mathbf{k}}^{0}}}
$$

in perfect agreement with Ref. 14 .

\section{Condensate density}

Taking now the derivative of the energy of Eq. (2.20) with respect to $\Gamma$, we obtain the following expression for the transverse magnetization:

$$
\begin{aligned}
m_{\perp}(S)= & S \sin \theta_{0} \\
& -\frac{1}{2 \sin \theta_{0}} \frac{1}{N} \sum_{\mathbf{k}}\left[\frac{A_{\mathbf{k}}^{0}}{\sqrt{\left(A_{\mathbf{k}}^{0}\right)^{2}-\left(B_{\mathbf{k}}^{0}\right)^{2}}}-1\right] \\
& -\frac{\cos ^{2} \theta_{0}}{4 \sin \theta_{0}} \frac{1}{N} \sum_{\mathbf{k}} \gamma_{\mathbf{k}} \sqrt{\frac{A_{\mathbf{k}}^{0}-B_{\mathbf{k}}^{0}}{A_{\mathbf{k}}^{0}+B_{\mathbf{k}}^{0}}}
\end{aligned}
$$

The condensate density $\rho_{0}$, which is the number of bosons occupying the $\mathbf{k}=0$ mode per site $\rho_{0}=\left\langle a_{\mathbf{k}=0}^{\dagger} a_{\mathbf{k}=0}\right\rangle / N=$ $\sum_{i j}\left\langle S_{i}^{+} S_{j}^{-}\right\rangle / N^{2}$, is simply related to the transverse magnetization by $\rho_{0}=\left[m_{\perp}(S=1 / 2)\right]^{2}$, which leads to the expression

$$
\begin{aligned}
\rho_{0}= & \frac{1}{4} \sin ^{2} \theta_{0}-\frac{1}{2 N} \sum_{\mathbf{k}}\left[\frac{A_{\mathbf{k}}^{0}}{\sqrt{\left(A_{\mathbf{k}}^{0}\right)^{2}-\left(B_{\mathbf{k}}^{0}\right)^{2}}}-1\right] \\
& -\frac{\cos ^{2} \theta_{0}}{4} \frac{1}{N} \sum_{\mathbf{k}} \gamma_{\mathbf{k}} \sqrt{\frac{A_{\mathbf{k}}^{0}-B_{\mathbf{k}}^{0}}{A_{\mathbf{k}}^{0}+B_{\mathbf{k}}^{0}}}
\end{aligned}
$$

This expression is different from that of Ref. 14. The two expressions are strictly equivalent only in the limit $\mu \rightarrow-4 t$ (low-density limit) and at $\mu=0$ (half-filling). In the range $-4<\mu / t<0$ and $0<\mu / t<4$, the expression of Ref. 14 differs form that of Eq. (2.28) by a term which is of order $1 / S^{2}$ [note that this difference comes from the fact that in Ref. 14 both the condensate and superfluid densities are expressed in terms of $\rho(1-\rho)$, where $\rho$ denotes the particle density corrected to order $1 / S .{ }^{16}$ We believe that the above expression for the transverse magnetization is the correct one to order $1 / S$. This will be further supported by a direct calculation of the expectation value of $S^{x}$ in the next section.

\section{Superfluid density}

As explained in Appendix A, the superfluid density is given at $1 / S$ order by the following expression:

$$
\begin{aligned}
\rho_{\mathrm{sf}}= & \frac{1}{4} \sin ^{2} \theta_{0}+\frac{1}{4 N t} \sum_{\mathbf{k}}\left[2 t-\sqrt{\left(A_{\mathbf{k}}^{0}\right)^{2}-\left(B_{\mathbf{k}}^{0}\right)^{2}}\right] \\
& -\frac{\cos ^{2} \theta_{0}}{4} \frac{1}{N} \sum_{\mathbf{k}} \gamma_{\mathbf{k}} \sqrt{\frac{A_{\mathbf{k}}^{0}-B_{\mathbf{k}}^{0}}{A_{\mathbf{k}}^{0}+B_{\mathbf{k}}^{0}}}
\end{aligned}
$$

This expression is equivalent to order $1 / S$ to the expression of Ref. 14. Looking at the expressions (2.28) and (2.29) for the condensed and superfluid densities, one can make several interesting observations. First, quantum fluctuations 
deplete the condensate whereas they enhance superfluidity. In Eq. (2.29), two contributions in the mechanism of superfluidity enhancement are present: the first term comes from the nearestneighbor kinetic energy, which increases (in absolute value) due to quantum fluctuations [see Eq. (2.20)], and the second one is due to the increase of the total density of particle $\rho$ as seen in Eq. (2.27). Interestingly, the same term appears in the condensate density, but with an opposite sign.

\section{LARGE $S$ CORRECTIONS TO THE HARMONIC GROUND STATE}

Now that we have expressions for the longitudinal and transverse magnetizations valid to order $1 / S$, let us show how these expressions can be obtained as expectation values of $S^{z}$ and $S^{x}$. Since the expressions to order $1 / S$ have been derived from the energy calculated at the harmonic level, one might expect that it is sufficient to calculate the expectation value of $S^{z}$ and $S^{x}$ in the harmonic ground state. As we shall see, this is not the case. The basic reason is quite simple: in terms of Holstein-Primakoff bosons, the operators $S^{z}$ and $S^{x}$ contains terms of order $O(S)$ and terms of order $O(1)$. To get an expression that is correct up to order $O(1)$, i.e., which includes all corrections up to $1 / S$, one should thus include in the ground-state corrections up to order $1 / \sqrt{S}$, if any, since the expectation value of the $O(S)$ part of the operators in such a correction will give a contribution of order $O(1)$. As we shall now show, the term $\mathcal{H}^{(3)}$ in the expansion of the Hamiltonian indeed leads to a correction to the ground state of order $1 / \sqrt{S}$.

\section{A. Beyond the harmonic ground state}

The objective of this part is to compute the large $S$ corrections to the harmonic ground state. To do so, we treat $\mathcal{H}^{(3)}$ (2.11) as a perturbation to $\mathcal{H}^{(2)}$, the small parameter being $1 / S$. The ground state of $\mathcal{H}^{(2)}$, the vacuum of $\alpha$ quasiparticles, being nondegenerate, we use Rayleigh-Schrödinger nondegenerate perturbation theory. To first order, the perturbed ground state is given by

$$
\begin{aligned}
|\psi\rangle & =|0\rangle+\sum_{|e\rangle} \underbrace{\frac{1}{E_{|0\rangle}-E_{|e\rangle}}}_{\mathcal{O}(S)} \underbrace{\left\langle e\left|\mathcal{H}^{(3)}\right| 0\right\rangle|e\rangle}_{\mathcal{O}\left(\frac{1}{s \sqrt{S}}\right)} \\
& =|0\rangle+\frac{1}{\sqrt{S}}\left|\phi^{\frac{1}{2}}\right\rangle+\mathcal{O}\left(\frac{1}{S}\right),
\end{aligned}
$$

where $|0\rangle$ denotes the vacuum of $\alpha$ quasiparticles, $|e\rangle$ magnon excitations and $E_{|0\rangle}\left(E_{|e\rangle}\right)$ the energy of the vacuum (excited states). The first correction to the ground state is of order $1 / \sqrt{S}$ since $1 /\left(E_{|0\rangle}-E_{|e\rangle}\right)$ is of order $\mathcal{O}(S)$, while $\left\langle e\left|\mathcal{H}^{(3)}\right| 0\right\rangle|e\rangle$ is of order $\mathcal{O}\left(1 / S^{\frac{3}{2}}\right)$. The second line defines $\left|\phi^{\frac{1}{2}}\right\rangle$, the ket that gives the $1 / \sqrt{S}$ correction to the ground state. The above wave function is correct to order $1 / \sqrt{S}$ since all terms $\mathcal{H}^{(n)}$ with $n \geqslant 4$ in the Holstein-Primakoff expansion (2.9) will contribute corrections of higher order in $1 / S$. Finally, we have to normalize the state, which leads to

$$
\left|\psi_{0}\right\rangle \approx\left(1-\frac{C}{2 S}\right)|0\rangle+\frac{1}{\sqrt{S}}\left|\phi^{\frac{1}{2}}\right\rangle+\cdots,
$$

where $C=\left\langle\phi^{\frac{1}{2}} \mid \phi^{\frac{1}{2}}\right\rangle$ and $\left\langle\psi_{0} \mid \psi_{0}\right\rangle=1+\mathcal{O}\left(1 / S^{2}\right)$. To compute $\left|\phi^{\frac{1}{2}}\right\rangle$, we first express $\mathcal{H}^{(3)}$ in Fourier space:

$$
\mathcal{H}^{(3)}=\frac{2 t}{S \sqrt{2 N S}} \sum_{\mathbf{k}, \mathbf{q}} \sin \theta \cos \theta \gamma_{\mathbf{q}}\left(b_{\mathbf{q}+\mathbf{k}}^{\dagger} b_{\mathbf{k}} b_{\mathbf{q}}+b_{\mathbf{k}-\mathbf{q}}^{\dagger} b_{\mathbf{k}} b_{\mathbf{q}}^{\dagger}\right)
$$

with

$$
\begin{aligned}
b_{\mathbf{q}+\mathbf{k}}^{\dagger} b_{\mathbf{k}} b_{\mathbf{q}}= & \left(u_{\mathbf{k}+\mathbf{q}} \alpha_{\mathbf{k}+\mathbf{q}}^{\dagger}-v_{\mathbf{k}+\mathbf{q}} \alpha_{-\mathbf{k}-\mathbf{q}}\right) \\
& \times\left(u_{\mathbf{k}} \alpha_{\mathbf{k}}-v_{\mathbf{k}} \alpha_{-\mathbf{k}}^{\dagger}\right)\left(u_{\mathbf{q}} \alpha_{\mathbf{q}}-v_{\mathbf{q}} \alpha_{-\mathbf{q}}^{\dagger}\right), \\
b_{\mathbf{k}-\mathbf{q}}^{\dagger} b_{\mathbf{k}} b_{\mathbf{q}}^{\dagger}= & \left(u_{\mathbf{k}-\mathbf{q}} \alpha_{\mathbf{k}-\mathbf{q}}^{\dagger}-v_{\mathbf{k}-\mathbf{q}} \alpha_{-\mathbf{k}+\mathbf{q}}\right) \\
& \times\left(u_{\mathbf{k}} \alpha_{\mathbf{k}}-v_{\mathbf{k}} \alpha_{-\mathbf{k}}^{\dagger}\right)\left(u_{\mathbf{q}} \alpha_{\mathbf{q}}^{\dagger}-v_{\mathbf{q}} \alpha_{-\mathbf{q}}\right) .
\end{aligned}
$$

$\mathcal{H}^{(3)}$ being a three-body operator, its effect on the vacuum is to create one-magnon or three-magnon excitations. In Sec. III B, we will show that, for the computation of first-order corrections to the average values of the observables of interest in this paper, only single magnon excitations are relevant. We thus write $\left|\phi^{\frac{1}{2}}\right\rangle$ as a sum of one-magnon and three-magnon contributions,

$$
\left|\phi^{\frac{1}{2}}\right\rangle=\left|\phi^{\frac{1}{2}}\right\rangle_{1 m}+\left|\phi^{\frac{1}{2}}\right\rangle_{3 m}
$$

and we concentrate on the expression of the one-magnon contribution $\left|\phi^{\frac{1}{2}}\right\rangle_{1 \mathrm{~m}}$. Due to momentum conservation in Eq. (3.3), the only single-particle excitations allowed have zero momenta. Hence $E_{|0\rangle}-E_{|e\rangle}=-\Omega_{0}=-2 \sqrt{A_{0}^{2}-B_{0}^{2}} / S$ and $\left|\phi^{\frac{1}{2}}\right\rangle_{1 m}$ is given by

$$
\begin{aligned}
\left|\phi^{\frac{1}{2}}\right\rangle_{1 m}= & -\frac{t}{\sqrt{2}} \sin \theta \cos \theta \frac{\left(u_{0}-v_{0}\right)}{\sqrt{A_{0}^{2}-B_{0}^{2}}} \\
& \times \frac{1}{\sqrt{N}} \sum_{\mathbf{k}}\left[2 v_{\mathbf{k}}^{2}+\gamma_{\mathbf{k}}\left(v_{\mathbf{k}}^{2}-v_{\mathbf{k}} u_{\mathbf{k}}\right)\right]\left|1_{\mathbf{q}=0}\right\rangle
\end{aligned}
$$

where $\left|1_{\mathbf{q}=0}\right\rangle$ denotes an excited state of one magnon with momenta $\mathbf{q}=0$. Note that it is only possible to write down such an expression because we have included a transverse field in the Hamiltonian, so that the Bogoliubov transformation is not singular at $\mathbf{k}=0$. This expression actually diverges in the limit $\Gamma \rightarrow 0$ because $\sqrt{A_{0}^{2}-B_{0}^{2}}=O\left(\Gamma^{1 / 2}\right)$, while $\left(u_{0}-\right.$ $\left.v_{0}\right)=O\left(\Gamma^{1 / 4}\right)$. As we shall see, the limit $\Gamma \rightarrow 0$ must be taken after calculating the expectation value of the operators.

\section{B. Expectation values of observables}

\section{Total density}

Let us first use the perturbed ground state to calculate the expectation value of $S^{z}$. The first step is to express $\left\langle S^{z}\right\rangle$ in terms of the spin operators in the rotated frame and to use the expansion of these operators in terms of Holstein-Primakoff 
bosons. This leads to

$$
\begin{aligned}
\left\langle S_{i}^{z}\right\rangle= & \cos \theta\left\langle S_{i}^{z^{\prime}}\right\rangle-\sin \theta\left\langle S_{i}^{x^{\prime}}\right\rangle \\
= & \cos \theta\left(S-\left\langle b_{i}^{\dagger} b_{i}\right\rangle\right) \\
& -\sin \theta\left\langle\frac{\sqrt{2 S}}{2}\left(b_{i}+b_{i}^{\dagger}\right)-\frac{1}{4 \sqrt{2 S}}\left(n_{i} b_{i}+b_{i}^{\dagger} n_{i}\right)\right\rangle .
\end{aligned}
$$

At the classical level, the average magnetization is given by $S \cos \theta_{0}$ when $\Gamma=0$. The spin-wave corrections to this result are of order $\mathcal{O}(1)$. Thus, given the structure of the perturbed ground state $\left|\psi_{0}\right\rangle \approx(1-C /(2 S))|0\rangle+S^{-\frac{1}{2}}\left|\phi^{\frac{1}{2}}\right\rangle$, the terms entering the average magnetization to order $\mathcal{O}(1)$ are

$$
\begin{aligned}
\left.\left\langle S_{i}^{z}\right\rangle\right|_{\Gamma=0}= & S \cos \theta_{0}-\lim _{\Gamma \rightarrow 0}\left[\cos \theta\left\langle 0\left|b_{i}^{\dagger} b_{i}\right| 0\right\rangle\right. \\
& \left.+\sin \theta \frac{1}{\sqrt{2}}\left(\left\langle 0\left|b_{i}^{\dagger}+b_{i}\right| \phi^{\frac{1}{2}}\right\rangle_{1 m}+\text { c.c. }\right)\right] .
\end{aligned}
$$

In the above expression, we have only included $\left|\phi^{\frac{1}{2}}\right\rangle_{1 m}$ in the matrix element of $b_{i}^{\dagger}+b_{i}$ since the operator $b_{i}^{\dagger}+b_{i}$ can at most create or destroy one Bogoliubov excitation. The threemagnon component $\left|\phi^{\frac{1}{2}}\right\rangle_{3 m}$ would only contribute to the matrix element $(1 / \sqrt{S})\left\langle 0\left|\left(n_{i} b_{i}+b_{i}^{\dagger} n_{i}\right) /(4 \sqrt{2 S})\right| \phi^{\frac{1}{2}}\right\rangle$, but this term is of order $\mathcal{O}(1 / S)$ and can be neglected since we are interested in the $O(1)$ correction to the expectation value of $S^{z}$.

The matrix elements $\left\langle 0\left|b_{i}^{\dagger} b_{i}\right| 0\right\rangle$ and $\left\langle 0\left|b_{i}^{\dagger}+b_{i}\right| \phi^{\frac{1}{2}}\right\rangle_{1 m}$ are readily computed in Fourier space:

$$
\left\langle 0\left|b_{i}^{\dagger} b_{i}\right| 0\right\rangle=\frac{1}{N} \sum_{\mathbf{k}} v_{\mathbf{k}}^{2}
$$

and

$$
\begin{aligned}
& \left\langle 0\left|b_{i}^{\dagger}+b_{i}\right| \phi^{\frac{1}{2}}\right\rangle_{1 m} \\
& =\frac{1}{\sqrt{N}} \sum_{\mathbf{k}^{\prime}}\left\langle 0\left|\left(b_{\mathbf{k}^{\prime}}^{\dagger} e^{-i \mathbf{k}^{\prime} \mathbf{r}_{i}}+b_{\mathbf{k}^{\prime}} e^{i \mathbf{k}^{\prime} \mathbf{r}_{i}}\right)\right| \phi^{\frac{1}{2}}\right\rangle_{1 m} \\
& =\frac{1}{\sqrt{N}}\left\langle 0\left|\left(u_{0}-v_{0}\right) \alpha_{0}\right| \phi^{\frac{1}{2}}\right\rangle_{1 m} \\
& =-\frac{t}{\sqrt{2}} \sin \theta \cos \theta \frac{1}{N} \sum_{\mathbf{k} \neq 0} \frac{2 v_{\mathbf{k}}^{2}+\gamma_{\mathbf{k}}\left(v_{\mathbf{k}}^{2}-v_{\mathbf{k}} u_{\mathbf{k}}\right)}{A_{0}+B_{0}}
\end{aligned}
$$

with

$$
A_{0}+B_{0}=2 t \sin ^{2} \theta_{0}+\Gamma\left(\frac{\cos ^{2} \theta_{0}}{\sin \theta_{0}}+\frac{1}{2 \sin \theta_{0}}\right)+\mathcal{O}\left(\Gamma^{2}\right) \text {. }
$$

Note that the expression of the second matrix element has a finite $\Gamma \rightarrow 0$ limit because of the extra $u_{0}-v_{0}$ factor. Injecting back Eqs. (3.10) and (3.11) into Eq. (3.9), one recovers exactly the expression of the longitudinal magnetization obtained before from the derivative of the energy calculated at the harmonic level.

\section{Condensate density}

The same procedure can be repeated for the operator $\left\langle S_{i}^{x}\right\rangle=$ $\sin \theta\left\langle S_{i}^{z^{\prime}}\right\rangle+\cos \theta\left\langle S_{i}^{x^{\prime}}\right\rangle$, and the $\Gamma \rightarrow 0$ limit of its expectation value is given by

$$
\begin{aligned}
\left.\left\langle S_{i}^{x}\right\rangle\right|_{\Gamma=0}= & S \sin \theta_{0}-\lim _{\Gamma \rightarrow 0}\left[\sin \theta\left\langle 0\left|b_{i}^{\dagger} b_{i}\right| 0\right\rangle\right. \\
& \left.-\cos \theta \frac{1}{\sqrt{2}}\left(\left\langle 0\left|b_{i}^{\dagger}+b_{i}\right| \phi^{\frac{1}{2}}\right\rangle_{1 m}+\text { c.c. }\right)\right] .
\end{aligned}
$$

Injecting back Eqs. (3.10) and (3.11) into this expression leads to exactly the same expression for the condensate as the one obtained from the derivative of the energy with respect to $\Gamma$.

\section{Momentum distribution}

The main advantage of this approach is that it gives access to observables that cannot be calculated as derivatives of the energy. Among them, a physically very important one is the momentum distribution defined by

$$
\left\langle a_{\mathbf{k}}^{\dagger} a_{\mathbf{k}}\right\rangle=\lim _{\substack{\Gamma \rightarrow 0 \\ S \rightarrow \frac{1}{2}}} \frac{1}{N} \sum_{i j}\left\langle S_{i}^{+} S_{j}^{-}\right\rangle e^{i \mathbf{k}\left(\mathbf{r}_{i}-\mathbf{r}_{j}\right)} .
$$

The details of the calculation of $\left\langle S_{i}^{+} S_{j}^{-}\right\rangle$in the perturbed ground state (3.2) are given in Appendix A. For $\mathbf{k} \neq 0$, the momentum distribution is given by

$$
\begin{aligned}
\left\langle a_{\mathbf{k}}^{\dagger} a_{\mathbf{k}}\right\rangle= & \frac{1}{4}\left(1+\cos \theta_{0}\right)^{2} \\
& +\frac{1}{2}\left[\left(1+\cos ^{2} \theta_{0}\right) v_{\mathbf{k}}^{2}+u_{\mathbf{k}} v_{\mathbf{k}} \sin ^{2} \theta_{0}\right] .
\end{aligned}
$$

The classical expression for $\left\langle a_{\mathbf{k}}^{\dagger} a_{\mathbf{k}}\right\rangle$ is equal to the square of the classical density and does not depend on k. Eq. (3.15) can be re-expressed as

$$
\left\langle a_{\mathbf{k}}^{\dagger} a_{\mathbf{k}}\right\rangle=\frac{2 t\left[1+\cos ^{2} \theta_{0}\left(1-\gamma_{\mathbf{k}}\right)\right]}{\Omega_{\mathbf{k}}}+\frac{\cos \theta_{0}}{2},
$$

from which one sees that the $1 / S$-corrected distribution diverges like $1 / \Omega_{\mathbf{k}}$ when approaching the condensate point at $\mathbf{k}=0$. More precisely, the momentum distribution is singular and behaves like $\sim \sin \theta_{0} / k$ for all values of the field $-4<$ $\mu / t<4$. The integral of this quantity over the whole Brillouin zone is convergent and yields the number of uncondensed particles, which is given by

$$
\begin{aligned}
\frac{1}{N} \sum_{\mathbf{k} \neq 0}\left\langle a_{\mathbf{k}}^{\dagger} a_{\mathbf{k}}\right\rangle= & \left(\rho^{\text {class }}\right)^{2}\left(1-\frac{1}{N}\right) \\
& +\frac{1}{N} \sum_{\mathbf{k} \neq 0} \frac{1}{2} \cos ^{2} \theta_{0}\left(v_{\mathbf{k}}^{2}-u_{\mathbf{k}} v_{\mathbf{k}}\right) \\
& +\frac{1}{N} \sum_{\mathbf{k} \neq 0} \frac{1}{2}\left(v_{\mathbf{k}}^{2}+u_{\mathbf{k}} v_{\mathbf{k}}\right) .
\end{aligned}
$$

Let us point out that one can also recover the condensate density using Eq. (B3) in Appendix A. Indeed, if $\mathbf{k}$ is replaced by zero in the right-hand side of Eq. (B3), the expression can be rearranged to lead again to the condensate density obtained previously using the definition $\rho_{0}=\left\langle S^{x}\right\rangle^{2}$.

\section{COMPARISON WITH QMC SIMULATIONS}

In this section, we compare the large $S$ approximation of various quantities to exact QMC estimates obtained using the 
stochastic-series-expansion (SSE) algorithm. ${ }^{25}$ The simulations have been performed for the hard-core boson model (1.1) on square lattices $L \times L$ with $L=8,16,24,32$ at temperatures low enough to get ground-state estimates $\left(\beta / t \propto L^{2}\right)$, in particular, in the dilute limit where the finite size gap scales as $\sim L^{-z}$ with $z=2$. Overall, we agree with the numerical results of Ref. 14 whenever we could compare. We have nevertheless included numerical results for the density, the condensate and the superfluid density to be able to discuss their behavior close to $\mu_{c}$, where the corrections to the harmonic ground state turn out to be crucial. In addition to these quantities that had already been discussed in Ref. 14, this section also contains $\mathrm{QMC}$ results for the momentum distribution.

\section{A. Particle density}

Figure 1 is a plot of the hardcore boson density as a function of $\mu / t$. The spin wave results for the density are plots of $\left\langle S^{z}\right\rangle+1 / 2$ calculated using the perturbed (solid line) and using the harmonic (dotted line) ground states. While both approaches yield significant corrections to the mean field density, the expectation value $\left\langle S^{z}\right\rangle$ calculated in the harmonic ground state misses some terms of order $O(1)$, i.e., $1 / S$ corrections to the classical results, as discussed in Sec. III B. This effect is best seen in the inset of Fig. 1, which shows the relative deviation of the spin-wave results from the QMC estimates. For small densities, the relative deviation from the QMC result is as large as $40 \%$ if $\left\langle S^{z}\right\rangle$ is computed using the harmonic ground state. This deviation never exceeds $5 \%$ if the perturbed ground state is used. Furthermore, the computation in the nonperturbed harmonic ground state misses a very important feature of the dilute Bose gas limit. Indeed, logarithmic corrections have been shown to dominate the low-density limit ${ }^{26-28}$ close to the critical point $\mu_{c}$, and QMC data are indeed consistent with the behavior $\rho \sim\left(\mu-\mu_{c}\right) \ln \left(\mu-\mu_{c}\right)$ with $\mu_{c}=-4 t$, as shown in Fig. 2. The density computed using the perturbed ground

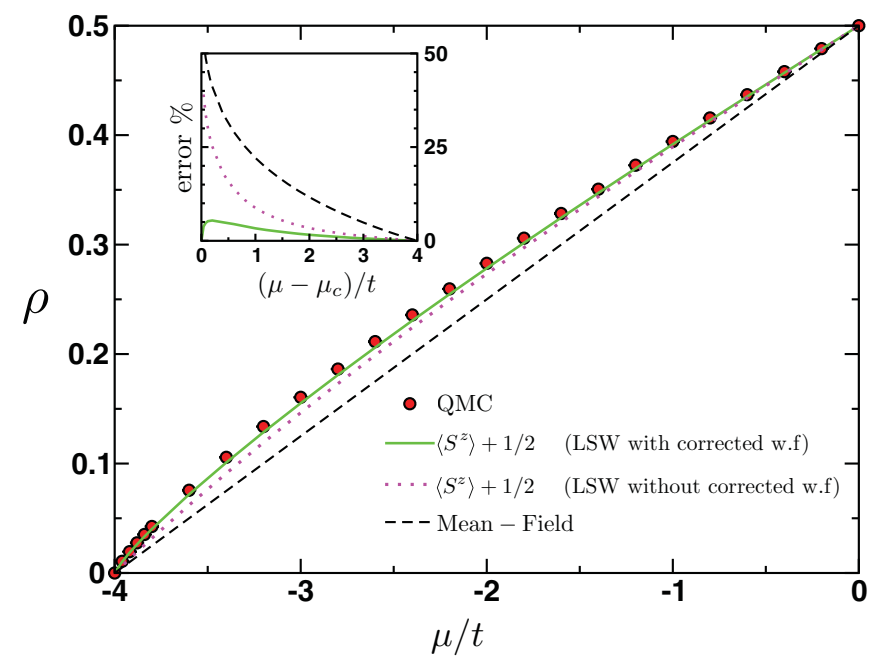

FIG. 1. (Color online) Hard-core boson density $\rho$ as a function $\mu / t$. QMC results (symbols) are compared with classical and LSW calculations. The inset shows the relative deviation of the classical and spin-wave results from the numerically exact QMC estimates.

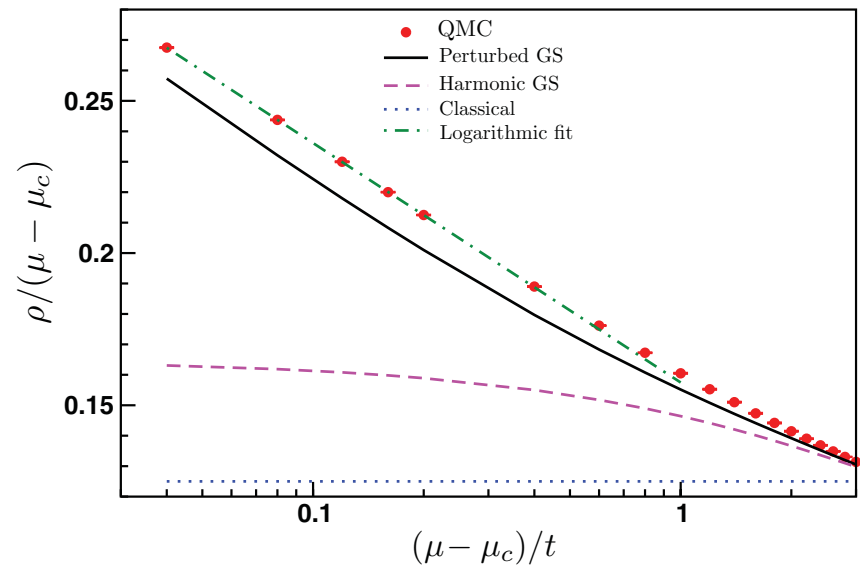

FIG. 2. (Color online) Logarithmic corrections to the density as a function of distance from the critical chemical potential $\left(\mu-\mu_{c}\right)$. QMC data (symbols) are described by a fit (dotted-dashed green line) of the form $\alpha\left|\ln \left[\epsilon\left(\mu-\mu_{c}\right) / 4 t\right]\right|$ with $\alpha \simeq 0.034$ and $\epsilon \simeq 0.04$; the spin-wave calculation using the perturbed ground state (black line) captures the logarithmic correction and yields $\alpha \simeq 0.035$ and $\epsilon \simeq 0.065$. Classical (blue dotted) and LSW results using the nonperturbed harmonic ground state (magenta dashed) do not capture the logarithmic corrections.

state correctly captures the logarithmic correction whereas $\left\langle S^{z}\right\rangle$ computed in the harmonic ground state does not.

These results show without any ambiguity that the best way to estimate the density in the context of a semiclassical approximation is to deduce it from the derivative of the harmonic energy with respect to the chemical potential, or equivalently to deduce it from a calculation of the expectation value of $S^{z}$ in the ground state that includes leading corrections beyond the harmonic approximation. The density deduced from the expectation value of $S^{z}$ calculated in the harmonic ground state is much less accurate, and qualitatively wrong in the dilute limit.

\section{B. Condensate density and superfluid density}

\section{LSW and QMC results}

Figures 3 and 4 are the plots of the condensate and superfluid densities as a function of $\mu / t$. The semiclassical results presented in the previous sections are in very good agreement with QMC results, as also discussed in Ref. 14. At the classical level, the condensate and the superfluid densities are equal. The effect of quantum fluctuations is to enhance the superfluidity and to deplete the condensate. QMC estimates for $\rho_{\text {sf }}$ and $\rho_{0}$ are obtained in the directed loop algorithm framework ${ }^{25}$ using the winding number fluctuations ${ }^{29}$ for $\rho_{\text {sf }}$ and the Green's function estimate ${ }^{30}$ for $\rho_{0}$. Note that the latter suffers from larger statistical errors than $\rho_{\text {sf }}$.

\section{Dilute Bose gas limit}

Building on the fact that the semiclassical results are very accurate, we analyze the extremely dilute limit for condensed and superfluid fractions using this approximate framework with the help of semiclassical calculations on finite square lattices of linear length $L=10^{5}$ down to very low particle 


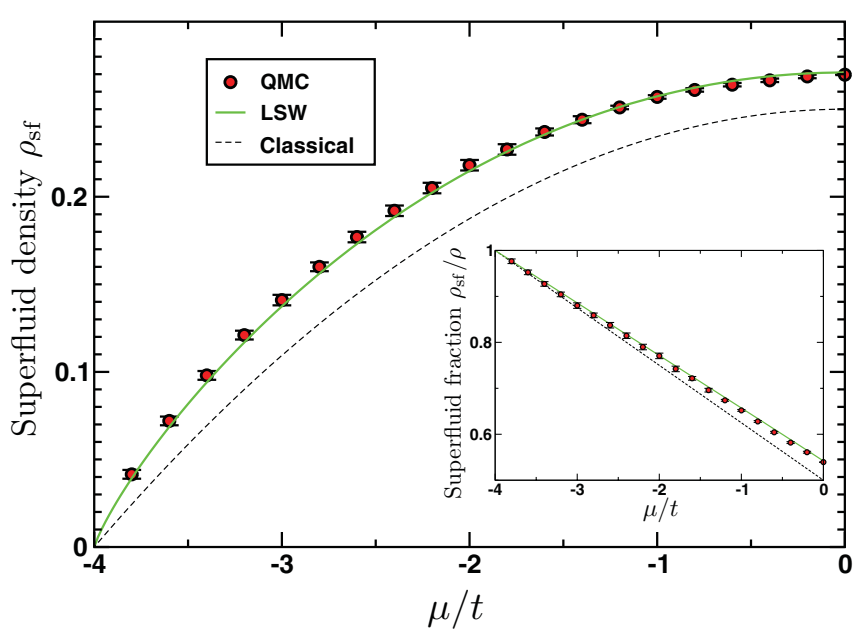

FIG. 3. (Color online) Superfluid density as a function of $\mu / t$. Classical (dashed line), semiclassical (solid green line), and QMC (symbols) results are shown. Inset: superfluid fraction.

density of $\rho=10^{-6}$. Such a limit is simply impossible to access using QMC simulations, where the computational cost grows very fast, like $\sim L^{4}$, so that only systems with a linear size of the order of $L \sim 10^{2}$ can be accessed. In both Figs. 3 and 4 , these fractions are shown in the insets. While both fractions are the same at the classical level, the effect of quantum fluctuations is qualitatively different in the two cases. In the extreme dilute limit, they converge to 1 very differently. Let us first consider the superfluid density. The superfluid fraction $\rho_{\text {sf }} / \rho$ is enhanced by quantum fluctuations with respect to the classical case, as seen in the inset of Fig. 3. More precisely, in the dilute limit, the semiclassical superfluid fraction (Fig. 5, left) tends to 1 like

$$
\rho_{\text {sf }} / \rho=1-\left(\zeta^{2} \rho\right)^{v},
$$

with $\zeta \simeq 0.728$, and an exponent $v \simeq 1.07$ very close to one. Note that, at the classical level, both fractions (superfluid and condensate) tend to 1 like $f=1-\rho$.

By contrast to the superfluid fraction, the condensed fraction is more affected by quantum fluctuations in the dilute limit. Indeed, it converges much more slowly to unity, as can be seen in the right panel of Fig. 5 and in the inset of Fig. 4. As first predicted by Schick in Ref. 26, logarithmic corrections of the form

$$
\rho_{0} / \rho=1-\frac{\alpha}{\left|\ln \left(\xi^{2} \rho\right)\right|}
$$

are expected in the extreme dilute limit. Figure 5 (right) shows semiclassical results for the very slow convergence of the condensate fraction to 1 , with a fit to Eq. (4.2) with $\alpha \simeq 0.86$ and $\xi \simeq 0.68$. In the theory of Ref. 26, the distance $\xi$ of Eq. (4.2) corresponds to the effective diameter of the hard core of the bosons, i.e., to the minimal distance between bosons. In our model, since bosons cannot be on the same site, but can occupy neighboring sites, we expect this effective minimal distance to be of the order of the lattice parameter (here equal to 1 ), and this is indeed the case since $\xi \simeq 0.68$. We also note that the same is true for the length scale of Eq. (4.1), and that the two length scales are of the same order $(\zeta \sim \xi \sim 0.7)$, a point not addressed in Ref. 26.

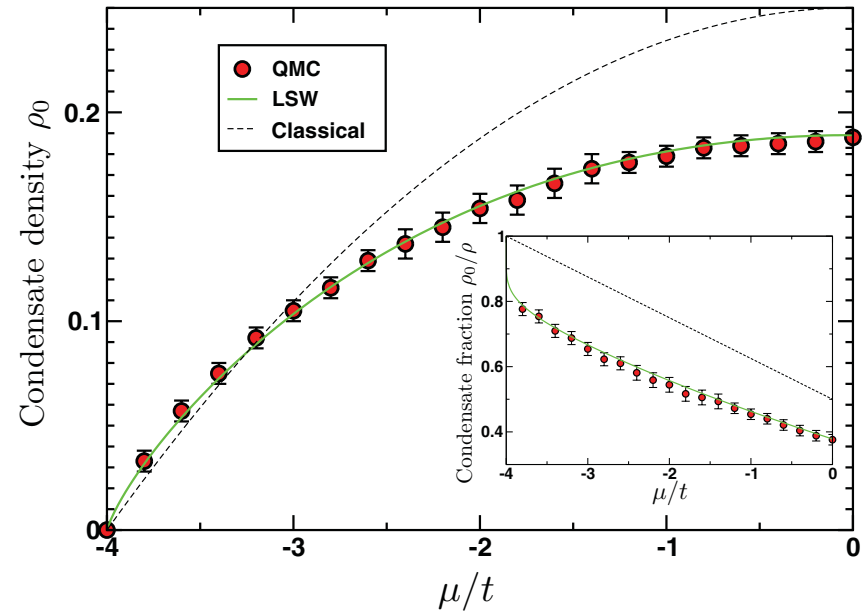

FIG. 4. (Color online) Condensate density as a function of $\mu / t$; classical (dashed line), semiclassical (solid green line), and QMC (symbols) results are shown. Inset: condensate fraction.

\section{Momentum distribution}

We now turn to the momentum distribution

$$
N(\mathbf{k})=\left\langle a_{\mathbf{k}}^{\dagger} a_{\mathbf{k}}\right\rangle,
$$

which can be efficiently computed using QMC simulations, following Ref. 30. Results for the half-filled case $(\mu=0)$ are shown in Fig. 6 for $k \neq 0$ along the line $k_{x}=k_{y}=k$ in the first Brillouin zone. At the classical level (dashed line), the distribution does not depend on momentum and is equal to $\left(\rho^{\text {class }}\right)^{2}$. The effect of spin-wave fluctuations (solid line) is to introduce a momentum dependence that is singular near $k=0$ and diverges like $1 / k$, as discussed in Sec. III B. This redistribution of spectral weight is due to the fact that spin waves deplete the condensate at $k=0$. The semiclassical calculation of the momentum distribution reproduces the behavior of the QMC results for small $k$. This is best seen in the right inset of the figure, which is a log-log
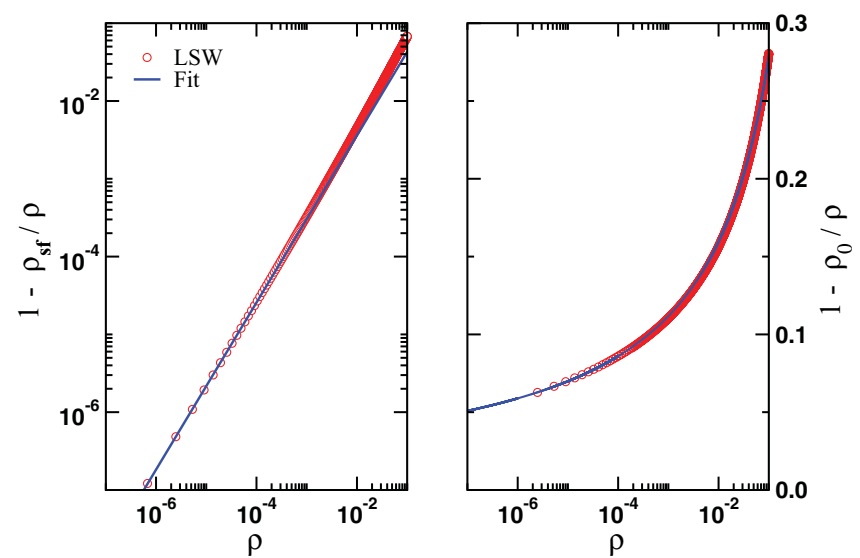

FIG. 5. (Color online) Semiclassical results (red symbols) for the superfluid (left) and condensate (right) fractions plotted versus the total density $\rho$. Blue lines are fits of the form Eq. (4.1) for the superfluid (left) and Eq. (4.2) for the condensate (right). 


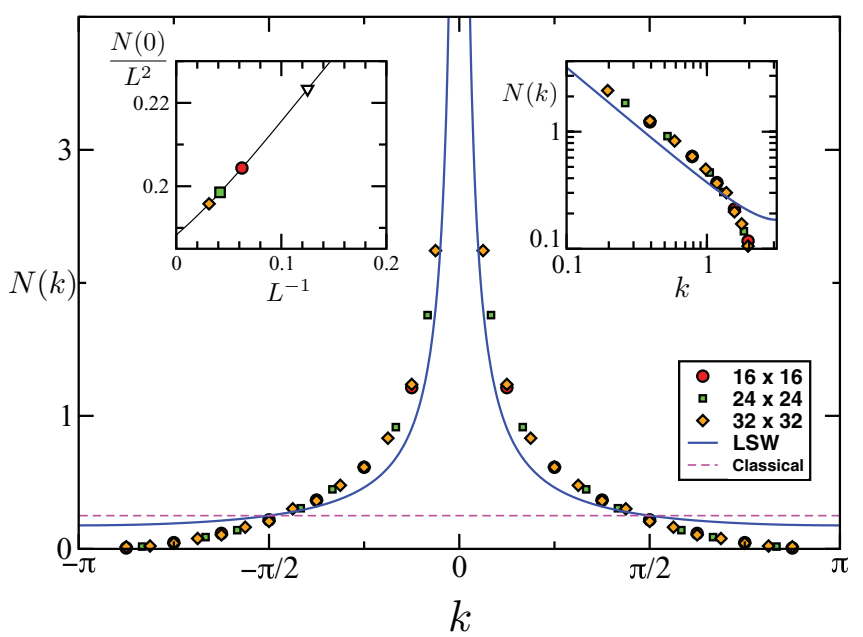

FIG. 6. (Color online) Momentum distribution of hard-core bosons $N(\mathbf{k}) \mathrm{Eq}(4.3)$ at half-filling $(\mu=0)$ along the line $k_{x}=$ $k_{y}=k$ for $k \neq 0$. Classical (dashed line), semiclassical (full line), and QMC (different symbols for $L=16,24,32$ ) results are shown together. Right inset: log-log plot of the momentum distribution, which diverges as $\sim 1 / k$. Left inset: finite size scaling of the condensate density from QMC as a function of $1 / L$. It is well accounted for by a quadratic fit (black line).

plot of the distribution showing the $1 / k$ dependence of the QMC results for small $k$. Looking back at Eq. (3.16), the $1 / k$ divergence of $N(k)$ is a consequence of the linear spectrum at small momentum $\Omega_{k} \sim k$. Away from the condensation vector $k=0$, the agreement between semiclassical and QMC results is less good. The QMC estimate is consistent with a distribution that goes to zero when $k \rightarrow \pi$ while, according to the semiclassical results, the distribution is only slightly renormalized downward with respect to the classical constant value.

The contribution at $k=0$ is not shown on the main panel of Fig. 6 since it diverges with the system size like $L^{2}$. In the left inset, however, we show the QMC result for $\rho_{0}=$ $N(0) / L^{2}$ plotted against $1 / L$. Using a quadratic fit, we extract the thermodynamic limit value of the condensate density at half-filling $\rho_{0} 0.188(2)$, which is in a good agreement with the estimate 0.191(2) reported by Sandvik and Hamer in Ref. 31 (see also Table I).

\section{DENSITY SUM RULE}

Having obtained the first-order corrections to the total density of particles, the density of condensed particles and the density of uncondensed particles, it is natural to test the semiclassical approximation with respect to the following sum rule:

$$
\rho=\rho_{0}+\frac{1}{N} \sum_{\mathbf{k} \neq 0} a_{\mathbf{k}}^{\dagger} a_{\mathbf{k}} .
$$

The above equality follows directly from the conservation of the number of particles: the total number of hardcore bosons in the system is equal to the sum of the number of condensed and uncondensed particles. Somewhat surprisingly, with the

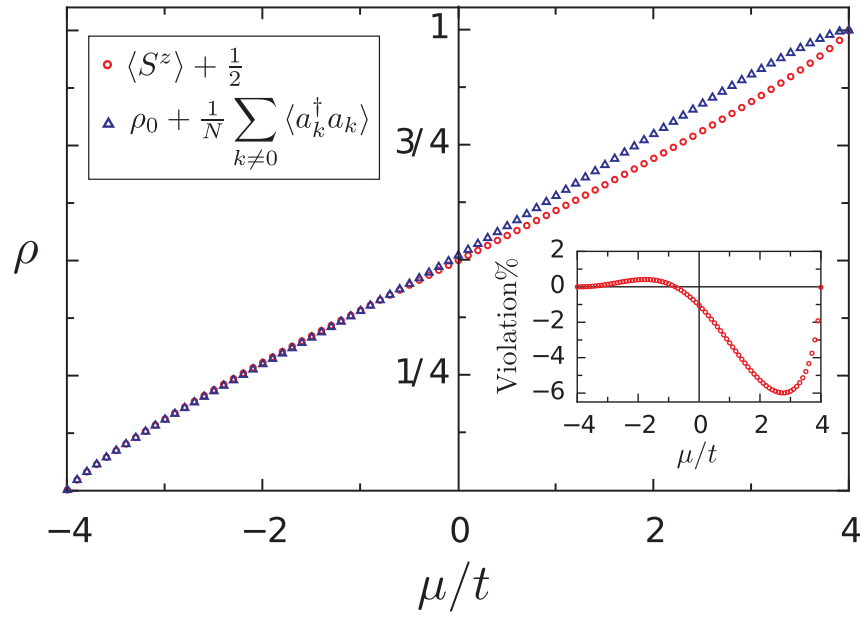

FIG. 7. (Color online) Total density $\rho$ (red diamonds) and $\rho_{0}+$ $\frac{1}{N} \sum_{\mathbf{k} \neq 0} a_{\mathbf{k}}^{\dagger} a_{\mathbf{k}}$ (blue triangles) as a function of the chemical potential. The inset presents the violation to the sum rule.

semiclassical expressions derived in the previous section, the sum rule of Eq. (5.1) is violated. Figure 7 shows the average particle density obtained from Eq. (2.27) and a plot of the sum $\rho_{0}+\left(\sum_{\mathbf{k} \neq 0} a_{\mathbf{k}}^{\dagger} a_{\mathbf{k}}\right) / N$ [the sum of Eqs. (2.28) and (3.17)]. In the inset, the violation of the sum rule, defined as the relative difference between the two quantities, is shown in the entire filling range. It is smaller than $2 \%$ up to half-filling, and never exceeds $6.5 \%$ above half-filling.

The origin of the violation of the sum rule is actually quite simple. In the spin-1/2 language, the sum rule relies on two identities:

$$
\sum_{i} S_{i}^{+} S_{i}^{-}=\frac{1}{N} \sum_{i, j} \sum_{\mathbf{k}} S_{i}^{+} S_{j}^{-} e^{i \mathbf{k}\left(\mathbf{r}_{i}-\mathbf{r}_{j}\right)}
$$

and

$$
S_{i}^{z}+1 / 2=S_{i}^{+} S_{i}^{-}
$$

which lead to

$$
\underbrace{\frac{1}{N} \sum_{i} n_{i}}_{\rho}=\underbrace{\frac{1}{N^{2}} \sum_{i, j} S_{i}^{+} S_{j}^{-}}_{\rho_{0}}+\underbrace{\frac{1}{N^{2}} \sum_{\substack{i, j \\ \mathbf{k} \neq 0}} S_{i}^{+} S_{j}^{-} e^{i \mathbf{k}\left(\mathbf{r}_{i}-\mathbf{r}_{j}\right)}}_{\frac{1}{N} \sum_{\mathbf{k} \neq 0} a_{\mathbf{k}}^{\dagger} a_{\mathbf{k}}}
$$

since, according to the Matsubara-Matsuda transformation, the local density is related to the $z$ component of the spin by $n_{i}=S_{i}^{z}+1 / 2$. However, when the spin is larger than $1 / 2$, the identity $S_{i}^{z}+1 / 2=S_{i}^{+} S_{i}^{-}$is no longer valid, and the expectation value of $S_{i}^{z}$ is no longer equal to that of $S_{i}^{+} S_{i}^{-}-1 / 2$.

Remarkably enough, in spite of that, the sum rule is almost satisfied, especially below half-filling. This provides some additional confidence in the accuracy of the semiclassical approach. As a further test, we discuss in the next section the effect of higher-order corrections on the ground-state energy. 


\section{SECOND-ORDER SPIN-WAVE THEORY}

\section{A. Energy}

The convergence of the expansion of spin operators in terms of Holstein-Primakoff bosons Eq. (2.8) can be a cause of concern in the case $S=1 / 2$. In this section, we compute the $1 / S^{2}$ correction to the expectation value of several observables.

The ground-state energy of the Hamiltonian to $\mathcal{H}^{(2)}$ gives the energy of the original problem to order $1 / S$. The $1 / S^{2}$ correction comes from both $\mathcal{H}^{(3)}$ and $\mathcal{H}^{(4)}$. It is obtained by treating $\mathcal{H}^{(3)}$ up to second order in perturbation theory, and $\mathcal{H}^{(4)}$ to first order, as pointed out in another context by Zhitomirsky and Nikuni. ${ }^{32}$ The contribution of $\mathcal{H}^{(4)}$ is simply given by $E^{(4)}=\left\langle\mathcal{H}^{(4)}\right\rangle / N$, where the expectation value is calculated in the harmonic ground state. Using Wick's theorem, this contribution is given by

$$
\begin{aligned}
E^{(4)}= & \lim _{\Gamma \rightarrow 0} \frac{t}{2 S^{2}}\left[\cos ^{2} \theta(2 m-\delta)(n-\Delta)\right. \\
& \left.+(2 m+\delta)(\Delta+n)-\sin ^{2} \theta\left(4 m^{2}+\Delta^{2}+n^{2}\right)\right],
\end{aligned}
$$

where $m, n, \delta$, and $\Delta$ are defined by

$$
\begin{aligned}
& m=\frac{1}{N} \sum_{\mathbf{k}} v_{\mathbf{k}}^{2}=\left\langle b_{i}^{\dagger} b_{i}\right\rangle, \\
& n=\frac{1}{N} \sum_{\mathbf{k}} v_{\mathbf{k}}^{2} \gamma_{\mathbf{k}}=2\left\langle b_{i}^{\dagger} b_{j}\right\rangle, \\
& \delta=\frac{1}{N} \sum_{\mathbf{k}} u_{\mathbf{k}} v_{\mathbf{k}}=-\left\langle b_{i} b_{i}\right\rangle, \\
& \Delta=\frac{1}{N} \sum_{\mathbf{k}} u_{\mathbf{k}} v_{\mathbf{k}} \gamma_{\mathbf{k}}=-2\left\langle b_{i} b_{j}\right\rangle,
\end{aligned}
$$

where $i$ and $j$ are nearest neighbors.

Being odd in the number of bosonic operators, $\mathcal{H}^{(3)}$ contributes only at second order in nondegenerate perturbation theory:

$$
E^{(3)}=\frac{1}{N} \sum_{|e\rangle \neq|0\rangle} \frac{\left|\left\langle e\left|\mathcal{H}^{(3)}\right| 0\right\rangle\right|^{2}}{E_{|0\rangle}-E_{|e\rangle}} \sim \mathcal{O}\left(\frac{1}{S^{2}}\right),
$$

where $E^{(3)}$ is the energy per site. The effect of $\mathcal{H}^{(3)}$ on the Bogoliubov vacuum is to create either single-magnon excited states or three-magnon excited states. We treat these two cases independently and write the $\mathcal{H}^{(3)}$ contribution to the energy as $E^{(3)}=E_{1 \mathrm{~m}}^{(3)}+E_{3 \mathrm{~m}}^{(3)}$. The single magnon component of $\mathcal{H}^{(3)}|0\rangle$ is

$$
\frac{2 t \sin \theta \cos \theta}{S \sqrt{2 S} \sqrt{N}} \sum_{\mathbf{k}}\left(u_{0}-v_{0}\right)\left[2 v_{\mathbf{k}}^{2}+\gamma_{\mathbf{k}}\left(v_{\mathbf{k}}^{2}-u_{\mathbf{k}} v_{\mathbf{k}}\right)\right]\left|1_{\mathbf{q}=0}\right\rangle,
$$

which leads to

$$
\begin{aligned}
E_{1 \mathrm{~m}}^{(3)}= & \lim _{\Gamma \rightarrow 0} \frac{-t^{2}}{S^{2}} \sin ^{2} \theta \cos ^{2} \theta \frac{\left(u_{0}-v_{0}\right)^{2}}{\sqrt{A_{0}^{2}-B_{0}^{2}}} \\
& \times \frac{1}{N^{2}}\left|\sum_{\mathbf{k}}\left[2 v_{\mathbf{k}}^{2}+\gamma_{\mathbf{k}}\left(v_{\mathbf{k}}^{2}-u_{\mathbf{k}} v_{\mathbf{k}}\right)\right]\right|^{2} \\
= & -\frac{t}{2 S^{2}} \cos ^{2} \theta_{0} \lim _{\Gamma \rightarrow 0}(2 m+n-\Delta)^{2}
\end{aligned}
$$

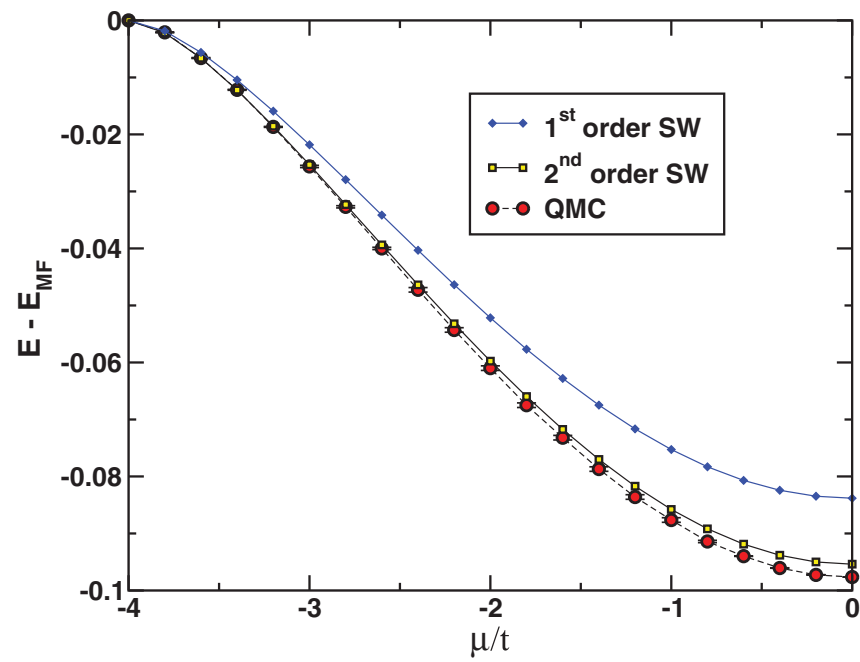

FIG. 8. (Color online) Plot of the first-order spin-wave energy, second-order spin-wave energy, and QMC energy measured with respect to the mean-field energy.

In the thermodynamic limit, $E_{3 \mathrm{~m}}^{(3)}$ is dominated by the excited states in which the three magnons all have different momenta. The three-magnon component of $\mathcal{H}^{(3)}|0\rangle$ that fulfills this condition is given by

$$
\begin{aligned}
& \frac{2 t \sin \theta \cos \theta}{S \sqrt{2 S} \sqrt{N}} \sum_{\mathbf{k}, \mathbf{q}} \mathrm{f}(\mathbf{q}, \mathbf{k})\left|1_{\mathbf{k}} 1_{\mathbf{q}} 1_{-\mathbf{k}-\mathbf{q}}\right\rangle, \\
& \mathrm{f}(\mathbf{q}, \mathbf{k})=\gamma_{\mathbf{q}}\left(u_{\mathbf{k}+\mathbf{q}} v_{\mathbf{k}} v_{\mathbf{q}}-u_{\mathbf{k}} u_{\mathbf{q}} v_{\mathbf{k}+\mathbf{q}}\right),
\end{aligned}
$$

where the sum $\sum_{\mathbf{k}, \mathbf{q}}^{\prime}$ is such that the three momenta $\mathbf{k}, \mathbf{q}$, and $-\mathbf{k}-\mathbf{q}$ are all different. The three-magnon contribution to $E^{(3)}$ takes the form

$$
\begin{aligned}
E_{3 \mathrm{~m}}^{(3)}= & \frac{-2 t^{2}}{S^{3}} \frac{1}{N^{2}} \sin ^{2} \theta_{0} \cos ^{2} \theta_{0} \\
& \times \lim _{\Gamma \rightarrow 0} \frac{1}{3 !} \sum_{\mathbf{k}, \mathbf{q}} \frac{\mathrm{F}^{2}(\mathbf{k}, \mathbf{q})}{\Omega_{\mathbf{k}}+\Omega_{\mathbf{q}}+\Omega_{\mathbf{k}+\mathbf{q}}},
\end{aligned}
$$

where $F(\mathbf{k}, \mathbf{q})$ is defined by

$$
\begin{aligned}
\mathrm{F}(\mathbf{k}, \mathbf{q})= & \mathrm{f}(\mathbf{k}, \mathbf{q})+\mathrm{f}(-\mathbf{k}-\mathbf{q}, \mathbf{q})+\mathrm{f}(\mathbf{q}, \mathbf{k})+\mathrm{f}(\mathbf{q},-\mathbf{k}-\mathbf{q}) \\
& +\mathrm{f}(-\mathbf{k}-\mathbf{q}, \mathbf{k})+\mathrm{f}(\mathbf{k},-\mathbf{k}-\mathbf{q}) .
\end{aligned}
$$

The spin-wave approximation of the energy per site to order $\mathcal{O}\left(1 / S^{2}\right)$,

$$
E=\mathcal{E}+E^{(2)}+E_{1 \mathrm{~m}}^{(3)}+E_{3 \mathrm{~m}}^{(3)}+E^{(4)},
$$

is plotted in Fig. 8, together with the first-order SWT and QMC results, as a function of $\mu / t$. All energies are measured with respect to the classical energy $\mathcal{E}$. The difference with the classical energy is monotonously increasing (in absolute value) from the dilute limit up to half-filling, where the correction due to quantum fluctuations is the most important. Clearly, the $1 / S$ correction captures most the quantum correction, but the inclusion of $1 / S^{2}$ corrections leads to a significantly better agreement with QMC results. This systematic improvement 
TABLE I. Ground-state estimates at half-filling $(\mu=0)$ for the energy per site $e_{0}$, the superfluid density $\rho_{\text {sf }}$, the condensate density $\rho_{0}$, and the compressibility $\kappa$. The three first lines are analytical results from classical and spin waves at first $(1 / S \mathrm{SW})$ and second order $\left(1 / S^{2} \mathrm{SW}\right)$. Below are shown QMC estimates from SSE simulations obtained by Sandvik in Ref. 31 and in this work.

\begin{tabular}{lllll}
\hline \hline & \multicolumn{1}{c}{$E_{\mathrm{gs}}$} & \multicolumn{1}{c}{$\rho_{\mathrm{sf}}$} & $\rho_{0}$ & \multicolumn{1}{c}{$\kappa$} \\
\hline Classical & -1 & 0.25 & 0.25 & 0.125 \\
$1 / S$ SW & -1.08382 & 0.27095 & 0.18904 & 0.1075 \\
$1 / S^{2}$ SW & -1.09539 & 0.27198 & 0.19127 & 0.1053 \\
QMC (Ref. 31) & $-1.097648(4)$ & $0.2696(2)$ & $0.191(2)$ & $0.1048(1)$ \\
QMC (this work) & $-1.09764(1)$ & $0.2697(2)$ & $0.188(2)$ & $0.1048(1)$ \\
\hline \hline
\end{tabular}

upon including higher-order $1 / S$ corrections gives additional support to the semiclassical expansion.

\section{B. Other observables}

Superfluid and condensate densities as well as the compressibility $\kappa=\partial \rho / \partial \mu$ can also be calculated at order $1 / S^{2}$. The calculation is straightforward but cumbersome and, for simplicity, it is not reproduced here. The estimates that we have obtained at half-filling are listed in Table I, together with QMC results from the present work as well as from Ref. 31. For the ground-state energy and the compressibility, the agreement increases systematically from $1 / S^{0}$ (classical) to $1 / S^{2}$. For the energy, the relative error is $\sim 9 \%$ for the classical estimate, less than $1.3 \%$ including the $1 / S$ correction, and $\sim 0.2 \%$ including the $1 / S^{2}$ correction. For the condensate density, the result including the $1 / S$ correction is already within the error bars of QMC, and it is not clear whether including $1 / S^{2}$ correction leads to any improvement. For the superfluid density, both the results including corrections up to order $1 / S$ and $1 / S^{2}$ lie outside the error bars of QMC, and the result up to order $1 / S$ appears to be better than the result up to order $1 / S^{2}$. In any case, the improvement over the classical result is clear for all quantities.

\section{CONCLUSION}

The semiclassical approach to hard-core bosons on a lattice, which is based on a large $S$ approximation to the Matsubara-Matsuda spin-1/2 version of the Hamiltonian, has been revisited with a few questions in mind: what is the correct way to get the exact $1 / S$ correction to various observables? Can the method be extended to a more complete characterization of ground-state correlations? How good is the semiclassical approach in dealing with some of the subtleties of bosons in $2 \mathrm{D}$, for instance, the logarithmic corrections of the dilute limit? We have shown that to get the exact $1 / S$ correction to the ground-state expectation value of various observables, it is necessary to include corrections to the harmonic ground state, and we have explicitly shown how to include them for the density, the condensate, and the momentum distribution function, for which, to the best of our knowledge, we have provided the first semiclassical expression. By a careful comparison with QMC results, we have shown that, when it is done properly, the semiclassical expansion is remarkably accurate. In particular, we have shown that it reproduces the logarithmic corrections predicted a long time ago in the dilute limit as well as the divergence of the momentum distribution at $k=0$. We have further tested the reliability of the $1 / S$ results by looking at the density sum rule and at higher order corrections. Whichever way one looks at it, the semiclassical approach appears as a very accurate description of hard-core bosons on a lattice.

\section{ACKNOWLEDGMENTS}

We are grateful to George Batrouni for very useful discussions about the results of Ref. 14. This project has been supported by the Swiss National Fund and by MaNEP.

\section{APPENDIX A: COMPUTATION OF THE SUPERFLUID DENSITY}

\section{Classical value}

The superfluid density can be obtained by imposing a phase gradient $\Phi_{\mathbf{r}_{i}+\mathbf{e}}-\Phi_{\mathbf{r}_{i}}=\varphi$ to the system (e being the unit vector along the $x$ or $y$ axis of the lattice). At the classical level, this leads to the following energy cost per site:

$$
E(\varphi)-E(0)=t\left(\sin \theta_{0}\right)^{2} \varphi^{2}+O\left(\varphi^{4}\right) .
$$

Using the analogy introduced by Fisher, Barber, and Jastrow in Ref. 33 where the kinetic energy density of a superflow (density $\rho_{\text {sf }}$ and velocity $\left.v_{\text {sf }}\right)$ in one direction is $\delta E\left(v_{\text {sf }}\right)=$ $\frac{1}{2} m^{*} \rho_{\mathrm{sf}}\left(v_{\mathrm{sf}}\right)^{2}$, with $v_{\mathrm{sf}}=\left(\hbar / m^{*}\right) \varphi$. This gives for the superfluid density

$$
\rho_{\mathrm{sf}}=\frac{m^{*}}{2 \hbar^{2}} \Upsilon_{\mathrm{sf}}
$$

where $\Upsilon_{\text {sf }}=\partial^{2} E(\varphi) /\left.\partial \varphi^{2}\right|_{\varphi=0}$ is the helicity modulus, the effective mass is given by $2 m^{*} / \hbar^{2}=1 /(2 t)$, and the factor of 2 comes from the fact that the twist $\varphi$ has been introduced in both directions. Finally, we get at the classical level

$$
\rho_{\text {sf }}=\frac{\sin ^{2} \theta_{0}}{4} \text {. }
$$

Interestingly, we remark that condensate and superfluid densities are equal at this level of approximation.

\section{SW corrections}

In order to evaluate the SW corrections to the superfluid fraction, the phase gradient can be introduced directly on the bosonic operators

$$
\begin{aligned}
& a_{i}^{\dagger} \rightarrow a_{i}^{\dagger} \mathrm{e}^{i \Phi_{i}}, \\
& a_{i} \rightarrow a_{i} \mathrm{e}^{-i \Phi_{i}},
\end{aligned}
$$


which in term of equivalent spin operators translates into

$$
\begin{gathered}
S_{i}^{x} \rightarrow S_{i}^{x} \cos \Phi_{i}-S_{i}^{y} \sin \Phi_{i}, \\
S_{i}^{y} \rightarrow S_{i}^{x} \sin \Phi_{i}+S_{i}^{y} \cos \Phi_{i} .
\end{gathered}
$$

Therefore the rotation (2.7) becomes

$$
\begin{aligned}
S_{i}^{x} & =\left(\cos \theta S_{i}^{u}+\sin \theta S_{i}^{w}\right) \cos \Phi_{i}-S_{i}^{v} \sin \Phi_{i}, \\
S_{i}^{y} & =\left(\cos \theta S_{i}^{u}+\sin \theta S_{i}^{w}\right) \sin \Phi_{i}+S_{i}^{v} \cos \Phi_{i}, \\
S_{i}^{z} & =-\sin \theta S_{i}^{u}+\cos \theta S_{i}^{w} .
\end{aligned}
$$

In the new rotated frame, at the linear $\mathrm{SW}$ approximation the $X Y$ Hamiltonian now reads

$$
\begin{aligned}
\mathcal{H}^{(2)}(\varphi) & =2 \sum_{\mathbf{k}}\left[A_{\mathbf{k}}(\varphi)\left(a_{\mathbf{k}}^{\dagger} a_{\mathbf{k}}+a_{-\mathbf{k}}^{\dagger} a_{-\mathbf{k}}\right)\right. \\
& \left.+B_{\mathbf{k}}(\varphi)\left(a_{\mathbf{k}}^{\dagger} a_{-\mathbf{k}}^{\dagger}+a_{\mathbf{k}} a_{-\mathbf{k}}\right)\right],
\end{aligned}
$$

with

$$
A_{\mathbf{k}}(\varphi)=-\frac{t}{2} \cos \varphi\left[\left(1+\cos ^{2} \theta\right) \gamma_{\mathbf{k}}-4\right]
$$

and

$$
B_{\mathbf{k}}(\varphi)=\frac{t}{2} \cos \varphi\left(\sin ^{2} \theta\right) \gamma_{\mathbf{k}},
$$

where we used $S=1 / 2$ and where $\theta$ is fixed by the equation $\cos \theta=\mu /(4 t \cos \phi)$ imposed by the minimization of the classical energy. At order $\varphi^{2}$, we have of course $\cos \varphi \simeq 1-\varphi^{2} / 2$. Similarly, the condition on $\theta$ yields $\cos ^{2} \theta \simeq \cos ^{2} \theta_{0}\left(1+\varphi^{2}\right)$ and $\sin ^{2} \theta \simeq \sin ^{2} \theta_{0}-\cos ^{2} \theta_{0} \varphi^{2}$. Therefore we have up to the $\operatorname{order} \varphi^{2}$ :

$$
A_{\mathbf{k}}(\varphi)=A_{\mathbf{k}}(0)-\frac{\varphi^{2}}{2}\left[A_{\mathbf{k}}(0)+t \gamma_{\mathbf{k}} \cos ^{2} \theta_{0}\right]
$$

and

$$
B_{\mathbf{k}}(\varphi)=B_{\mathbf{k}}(0)-\frac{\varphi^{2}}{2}\left[B_{\mathbf{k}}(0)+t \gamma_{\mathbf{k}} \cos ^{2} \theta_{0}\right] .
$$

Writing $A_{\mathbf{k}}(0)=A_{\mathbf{k}}$ and $B_{\mathbf{k}}(0)=B_{\mathbf{k}}$, the $1 / S$ correction to the GS energy in the presence of a small twist reads

$$
\begin{aligned}
& E^{(2)}(\varphi)-E^{(2)}(0) \\
& \quad=\frac{\varphi^{2}}{N} \sum_{\mathbf{k}}\left(2 t-\sqrt{A_{\mathbf{k}}^{2}-B_{\mathbf{k}}^{2}}-t \gamma_{\mathbf{k}} \cos ^{2} \theta_{0} \sqrt{\frac{A_{\mathbf{k}}-B_{\mathbf{k}}}{A_{\mathbf{k}}+B_{\mathbf{k}}}}\right) .
\end{aligned}
$$

Thus the superfluid density is given at $1 / S$ order by the following expression:

$$
\begin{aligned}
\rho_{\mathrm{sf}}= & \frac{\sin ^{2} \theta_{0}}{4} \\
& +\frac{1}{4 N t} \sum_{\mathbf{k}}\left(2 t-\sqrt{A_{\mathbf{k}}^{2}-B_{\mathbf{k}}^{2}}\right. \\
& \left.-t \gamma_{\mathbf{k}} \cos ^{2} \theta_{0} \sqrt{\frac{A_{\mathbf{k}}-B_{\mathbf{k}}}{A_{\mathbf{k}}+B_{\mathbf{k}}}}\right) .
\end{aligned}
$$

This expression and that of Ref. 14 are strictly equivalent only at $\mu=-4 t$ (low-density limit) and at $\mu=0$ (half-filling). In the range $-4<\mu / t<0$ and $0<\mu / t<4$, they differ by a term which is of order $1 / S^{2}$.

\section{APPENDIX B: MOMENTUM DISTRIBUTION}

We start by expressing $S_{i}^{+} S_{j}^{-}$in the rotated frame:

$$
\begin{aligned}
S_{i}^{+} S_{j}^{-}= & \cos ^{2} \theta S_{i}^{x^{\prime}} S_{j}^{x^{\prime}}+\sin ^{2} \theta S_{i}^{z^{\prime}} S_{j}^{z^{\prime}}+S_{i}^{y^{\prime}} S_{j}^{y^{\prime}} \\
& +\cos \theta \sin \theta\left(S_{i}^{x^{\prime}} S_{j}^{z^{\prime}}+S_{i}^{z^{\prime}} S_{j}^{x^{\prime}}\right) \\
& +\cos \theta S_{i}^{z^{\prime}} \delta_{i, j}-\sin \theta S_{i}^{x^{\prime}} \delta_{i, j} .
\end{aligned}
$$

The terms involved in $\left\langle S_{i}^{+} S_{j}^{-}\right\rangle$up to order $\mathcal{O}(S)$ are

$$
\begin{aligned}
\left\langle S_{i}^{+} S_{j}^{-}\right\rangle= & \frac{S}{2} \cos ^{2} \theta\left\langle 0\left|\left(b_{i}+b_{i}^{\dagger}\right)\left(b_{j}+b_{j}^{\dagger}\right)\right| 0\right\rangle \\
& +S^{2} \sin ^{2} \theta-S \sin ^{2} \theta\left\langle 0\left|b_{i}^{\dagger} b_{i}+b_{j}^{\dagger} b_{j}\right| 0\right\rangle \\
& -\frac{S}{2}\left\langle 0\left|\left(b_{i}-b_{i}^{\dagger}\right)\left(b_{j}-b_{j}^{\dagger}\right)\right| 0\right\rangle \\
& +\cos \theta \sin \theta \frac{S}{\sqrt{2}}\left(\left\langle 0\left|\left(b_{i}+b_{i}^{\dagger}\right)\right| \phi^{\frac{1}{2}}\right\rangle+\text { c.c. }\right) \\
& +\cos \theta \sin \theta \frac{S}{\sqrt{2}}\left(\left\langle 0\left|\left(b_{j}+b_{j}^{\dagger}\right)\right| \phi^{\frac{1}{2}}\right\rangle+\text { c.c. }\right) \\
& +S \cos \theta \delta_{i, j} .
\end{aligned}
$$

Injecting this result in Eq. (3.14) and making use of the definitions of the inverse Fourier transforms of the $b_{i}$ operators, Eq. (2.13), we obtain

$$
\begin{aligned}
\left\langle a_{\mathbf{k}}^{\dagger} a_{\mathbf{k}}\right\rangle= & \lim _{\substack{\Gamma \rightarrow 0 \\
S \rightarrow \frac{1}{2}}}\left[\frac{S}{2} \cos ^{2} \theta\left\langle 0\left|\left(b_{-\mathbf{k}}+b_{\mathbf{k}}^{\dagger}\right)\left(b_{\mathbf{k}}+b_{-\mathbf{k}}^{\dagger}\right)\right| 0\right\rangle\right. \\
& +S^{2} \sin ^{2} \theta N \delta_{\mathbf{k}, 0} \\
& -S \sin ^{2} \theta \delta_{\mathbf{k}, 0}\left\langle 0\left|\sum_{i} b_{i}^{\dagger} b_{i}\left(e^{i \mathbf{k} r_{i}}+e^{-i \mathbf{k} r_{i}}\right)\right| 0\right\rangle \\
& -\frac{S}{2}\left\langle 0\left|\left(b_{-\mathbf{k}}-b_{\mathbf{k}}^{\dagger}\right)\left(b_{\mathbf{k}}-b_{-\mathbf{k}}^{\dagger}\right)\right| 0\right\rangle \\
& +\cos \theta \sin \theta \frac{\sqrt{N} S}{\sqrt{2}} \delta_{\mathbf{k}, 0}\left(|0|\left(b_{-\mathbf{k}}+b_{\mathbf{k}}^{\dagger}\right)\left|\phi^{\frac{1}{2}}\right\rangle+\text { c.c. }\right) \\
& +\cos \theta \sin \theta \frac{\sqrt{N} S}{\sqrt{2}} \delta_{\mathbf{k}, 0}\left(|0|\left(b_{\mathbf{k}}+b_{-\mathbf{k}}^{\dagger}\right)\left|\phi^{\frac{1}{2}}\right\rangle+\text { c.c. }\right) \\
& +S \cos \theta] .
\end{aligned}
$$

Hence the number of particles at momentum $\mathbf{k} \neq 0$ is given by

$$
\begin{aligned}
\left\langle a_{\mathbf{k}}^{\dagger} a_{\mathbf{k}}\right\rangle= & \lim _{\Gamma \rightarrow 0}\left[\frac{S}{2} \cos ^{2} \theta\left\langle 0\left|\left(b_{-\mathbf{k}}+b_{\mathbf{k}}^{\dagger}\right)\left(b_{\mathbf{k}}+b_{-\mathbf{k}}^{\dagger}\right)\right| 0\right\rangle\right. \\
& S \rightarrow \frac{1}{2} \\
& \left.-\frac{S}{2}\left\langle 0\left|\left(b_{-\mathbf{k}}-b_{\mathbf{k}}^{\dagger}\right)\left(b_{\mathbf{k}}-b_{-\mathbf{k}}^{\dagger}\right)\right| 0\right\rangle+S \cos \theta\right] \\
= & \frac{1}{4}\left(1+\cos \theta_{0}\right)^{2} \\
& +\left[\left(1+\cos ^{2} \theta_{0}\right) v_{\mathbf{k}}^{2}+u_{\mathbf{k}} v_{\mathbf{k}} \sin ^{2} \theta_{0}\right] / 2 .
\end{aligned}
$$


${ }^{1}$ H. M. Jaeger, D. B. Haviland, B. G. Orr, and A. M. Goldman, Phys. Rev. B 40, 182 (1989).

${ }^{2}$ R. Fazio and H. van der Zant, Phys. Rep. 355, 235 (2001).

${ }^{3}$ T. Masubara and H. Matsuda, Prog. Theoret. Phys. 16, 569 (1956).

${ }^{4}$ W. Krauth and N. Trivedi, Europhys. Lett. 14, 627 (1991).

${ }^{5}$ D. M. Ceperley, Rev. Mod. Phys. 67, 279 (1995).

${ }^{6}$ I. Bloch, J. Dalibard, and W. Zwerger, Rev. Mod. Phys. 80, 885 (2008).

${ }^{7}$ A. S. Alexandrov and N. F. Mott, Rep. Prog. Phys. 57, 1197 (1994).

${ }^{8}$ I. Affleck, Phys. Rev. B 43, 3215 (1991).

${ }^{9}$ T. Giamarchi, C. Rüegg, and O. Tchernyshyov, Nat. Phys. 4, 198 (2008).

${ }^{10}$ F. Hébert, G. G. Batrouni, R. T. Scalettar, G. Schmid, M. Troyer, and A. Dorneich, Phys. Rev. B 65, 014513 (2001).

${ }^{11}$ F. Mila and K. P. Schmidt, in Introduction to Frustrated Magnetism, edited by C. Lacroix, F. Mila, and P. Mendels, Springer Series in Solid-State Sciences Vol. 164 (Springer-Verlag, Berlin, Heidelberg, 2011), p. 537.

${ }^{12}$ R. T. Scalettar, G. G. Batrouni, A. P. Kampf, and G. T. Zimanyi, Phys. Rev. B 51, 8467 (1995).

${ }^{13}$ G. Murthy, D. Arovas, and A. Auerbach, Phys. Rev. B 55, 3104 (1997).
${ }^{14}$ K. Bernardet, G. G. Batrouni, J. L. Meunier, G. Schmid, M. Troyer, and A. Dorneich, Phys. Rev. B 65, 104519 (2002).

${ }^{15}$ C. Pich and E. Frey, Phys. Rev. B 57, 13712 (1998).

${ }^{16} \mathrm{G}$. Batrouni (private communication).

${ }^{17}$ N. Laflorencie and F. Mila, Phys. Rev. Lett. 102, 060602 (2009).

${ }^{18}$ R. Schaffer, A. A. Burkov, and R. G. Melko, Phys. Rev. B 80, 014503 (2009).

${ }^{19}$ H. T. Ueda and K. Totsuka, Phys. Rev. B 81, 054442 (2010).

${ }^{20}$ T. Durić and D. K. K. Lee, Phys. Rev. B 81, 014520 (2010).

${ }^{21}$ N. Laflorencie and F. Mila, Phys. Rev. Lett. 107, 037203 (2011).

${ }^{22}$ I. Hen and M. Rigol, Phys. Rev. B 80, 134508 (2009).

${ }^{23}$ I. Hen and M. Rigol, Phys. Rev. A 82, 043634 (2010).

${ }^{24}$ T. Holstein and H. Primakoff, Phys. Rev. 58, 1098 (1940).

${ }^{25}$ O. F. Syljuåsen and A. W. Sandvik, Phys. Rev. E 66, 046701 (2002).

${ }^{26}$ M. Schick, Phys. Rev. A 3, 1067 (1971).

${ }^{27}$ V. N. Popov, Theor. Math. Phys. 11, 565 (1972).

${ }^{28}$ D. S. Fisher and P. C. Hohenberg, Phys. Rev. B 37, 4936 (1988).

${ }^{29}$ E. L. Pollock and D. M. Ceperley, Phys. Rev. B 36, 8343 (1987).

${ }^{30}$ A. Dorneich and M. Troyer, Phys. Rev. E 64, 066701 (2001).

${ }^{31}$ A. W. Sandvik and C. J. Hamer, Phys. Rev. B 60, 6588 (1999).

${ }^{32}$ M. E. Zhitomirsky and T. Nikuni, Phys. Rev. B 57, 5013 (1998).

${ }^{33}$ M. E. Fisher, M. N. Barber, and D. Jasnow, Phys. Rev. A 8, 1111 (1973). 